\title{
An Efficient QoS MAC for IEEE802.11p Over Cognitive Multichannel Vehicular Networks
}

\author{
by \\ Hikmat El Ajaltouni \\ Thesis submitted to the \\ Faculty of Graduate and Postdoctoral Studies \\ In partial fulfillment of the requirements \\ For the M.A.Sc. degree in \\ Electrical and Computer Engineering
}

School of Information Technology and Engineering

Faculty of Engineering

University of Ottawa

(c) Hikmat El Ajaltouni, Ottawa, Canada, 2012 


\begin{abstract}
One of the most challenging issues facing vehicular networks lies in the design of an efficient MAC protocol due to mobile nature of nodes, delay constraints for safety applications and interference.

In this thesis, I propose an efficient Multichannel QoS Cognitive MAC (MQOG). MQOG assesses the quality of channel prior to transmission employing dynamic channel allocation and negotiation algorithms to achieve significant increase in channel reliability, throughput and delay constraints while simultaneously addressing Quality of Service. The uniqueness of MQOG lies in making use of the free unlicensed bands. To consider fair effective sharing of resources I propose a Mobility Based Dynamic Transmit Opportunity (MoByToP) while modifying the 802.11e TXOP (Transmit Opportunity).

The proposed protocols were implemented in OMNET ++4.1 , and extensive experiments demonstrated a faster and more efficient reception of safety messages compared to existing VANet MAC Protocols. Finally, improvements in delay, packet delivery ratios and throughput were noticed.
\end{abstract}




\section{Acknowledgements}

My profound thanks go in the first place to Prof. Azzedine Boukerche of the School of Information Technology and Engineering (S.I.T.E) at the University of Ottawa whom through his valuable advice, enduring patience and constructive criticism followed me step-by step through the progress of this thesis. Not only Prof. Azzedine was a great source of academic guidance but also he provided me with conditions that were conducive to prolific research. He was never ceasing in his belief in me, always providing clear explanations and always giving me his time. I wish all students the honor and opportunity to meet him and experience his academic ability.

Special thanks go to Dr. Richard W. Pazzi for guidance and encouragement in carrying out this thesis and never hesitating in answering all my questions from the first day till the last. His comments were highly appreciated and I am very glad to get to know such a person like Dr. Pazzi.

In addition, I would like to thank my colleagues at PARADISE Research Laboratory and NSERC DIVA Research center for their continuous support and collaboration. They gave all the best to help me finish this work. The lab meetings and the DIVA Seminars held broadened my knowledge in vehicular networking and gave me insights to come up with new contributions. No doubt all PARADISE and DIVA members are great people.

I would sincerely like to thank too my brother Elie El Ajaltouni who introduced me to Professor Boukerche. During my stay in Canada, Elie was always there to assist me and answer all my questions. He has always been a constant source of love, support and strength all those years.

Last but not least, I would like to thank my parents for their moral, financial and never ending support for without them I would never achieve what I did. THANK YOU ALL. 


\title{
Dedication
}

\author{
To my
}

MOTHER, FATHER, BROTHERS, and GRANDMOTHER

with love and appreciation 


\section{List of Publications}

The following publications are relevant to the topic of this thesis and have been authored by Hikmat El Ajaltouni.

\section{Conferences:}

1. Hikmat El Ajaltouni, Richard W. Pazzi, Azzedine Boukerche. An Efficient QoS MAC for IEEE802.11p Over Cognitive Multichannel Vehicular Networks. Accepted in IEEE International Conference On Communications (ICC), 2012 to be held in Ottawa,Canada in June 2012.

2. Hikmat El Ajaltouni, Richard W. Pazzi, Azzedine Boukerche. Mobility Based Dynamic TXOP for Vehicular Communications. Submitted to the 9th Annual IEEE Communications Society Conference on Sensor, Mesh and Ad Hoc Communications and Networks (SECON), 2012 to be held in Seoul,Korea in June 2012. 


\section{Contents}

1 Introduction $\quad 1$

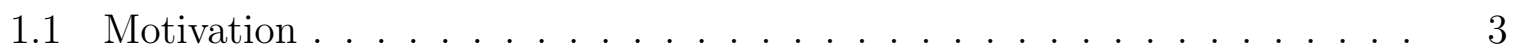

1.2 Thesis Objective . . . . . . . . . . . . . . . . . 4

1.3 Contribution . . . . . . . . . . . . . . . . . . 6

1.4 Thesis Organization . . . . . . . . . . . . . . . . . . . . 6

2 Related Work $\quad 8$

2.1 Background . . . . . . . . . . . . . . . . . 8

2.1.1 MAC: Medium Access Control . . . . . . . . . . . . . . . 8

2.1.2 IEEE 802.11 MAC Distributed Coordination Function (DCF) . . 11

2.2 Medium Access Control Design Guidelines . . . . . . . . . . . . . 13

2.3 Single Radio Single Channel Solutions . . . . . . . . . . . . . . . . . . 15

2.3.1 Space Division Multiple Access (SDMA) Solutions . . . . . . . . . 15

$2.3 .2 \mathrm{ADHOC} \mathrm{MAC} \ldots \ldots \ldots \ldots$

2.3.3 Repetition-based MAC . . . . . . . . . . . . . . 17

2.3.4 Directional Antenna-Based MAC . . . . . . . . . . . . . 18

2.4 Single Radio Multichannel Solutions . . . . . . . . . . . . . . . . . . . . . 19

2.4.1 IEEE802.11p Protocol . . . . . . . . . . . . . . . . . . . 19

2.4.2 VMESH: Distributed Segment Storage for Peer-to-Peer Streaming 23 
2.5 Multiradio Multichannel Solutions . . . . . . . . . . . . . . . . 24

2.5.1 Cluster Based MAC Solutions . . . . . . . . . . . . . . . . . 24

2.5.2 On-Demand Channel Assignment (DCA) for Multichannel MAC 25

2.6 Comparison of Existing Protocols . . . . . . . . . . . . . . 26

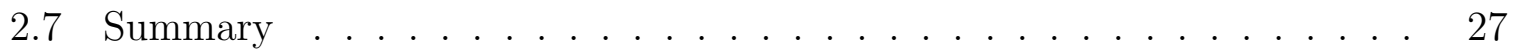

3 Proposed Multichannel QoS Cognitive MAC for VANets (MQOG) 28

3.1 Motivation . . . . . . . . . . . . . . . . . . . . 28

3.2 Cognitive Radios and Unlicensed Bands: Approach . . . . . . . . . . 29

3.3 Proposed System Architecture . . . . . . . . . . . . . . . . . 31

3.3.1 Proposed MAC Radio Architecture . . . . . . . . . . . . . . . . 32

3.3.2 Supported Frames and Tables in the Proposed Protocol . . . . . . 33

3.3 .3 Overall System Operation . . . . . . . . . . . . . . . . . 36

3.4 Dynamic Channel Allocation Algorithm . . . . . . . . . . . . . . . 37

3.5 Channel Negotiation Algorithm _. . . . . . . . . . . . . 40

3.6 Conclusion . . . . . . . . . . . . . . . . . . . . . . . . 42

4 Enhanced Multichannel QoS Cognitive MAC for VANets (EMQOG) 43

4.1 Motivation . . . . . . . . . . . . . . . . . 43

4.2 Transmit Opportunity (TXOP) and Related Work . . . . . . . . . . 46

4.2 .1 TXOP Defined . . . . . . . . . . . . . . 46

4.2 .2 Related Work Using TXOP . . . . . . . . . . . . . . . 47

4.3 A Proposed Dynamic TXOP (MoByToP): Design . . . . . . . . . . . 49

4.4 Enhanced-MQOG: Integrating MoByToP Scheme with MQOG . . . . . . 56

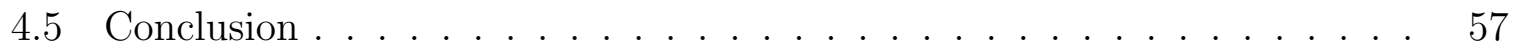

5 Performance Evaluation of Our Proposed MAC Protocols 58

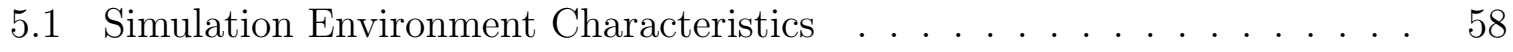


5.2 Simulation Parameters, Process and Metrics . . . . . . . . . . . . 62

5.3 Performance Evaluation of the Proposed Multichannel MAC . . . . . . . 64

5.3.1 Simulation Scenario . . . . . . . . . . . . . . . . 64

5.3 .2 Results and Interpretation . . . . . . . . . . . . 65

5.4 Performance Evaluation of the Proposed Enhanced Multichannel MAC . 70

5.4 .1 Simulation Scenario . . . . . . . . . . . . 70

5.4 .2 Results and Interpretation . . . . . . . . . . . . . 71

5.5 Conclusion . . . . . . . . . . . . . . . . . 77

6 Conclusion and Future Work $\quad 78$

6.1 Future Work . . . . . . . . . . . . . . . . 79 


\section{List of Tables}

1.1 Examples of DSRC Applications and Requirements[5] . . . . . . . . . . 4

2.1 Comparison of Different MAC Protocols . . . . . . . . . . . . 26

3.1 QoS Different Category Levels . . . . . . . . . . . . . . . . . . . 36

3.2 Channel Assessment Properties [8] . . . . . . . . . . . . . . . . . . . . 39

$4.1 \quad 802.11$ e EDCA Parameter Settings . . . . . . . . . . . . . . 47

$5.1 \quad$ Map Areas . . . . . . . . . . . . . . . . . . . . . . . . 59

5.2 General Simulation Parameters . . . . . . . . . . . . . . 63

5.3 Scenario Types . . . . . . . . . . . . . . . . . . . 71 


\section{List of Figures}

2.1 OSI Model . . . . . . . . . . . . . . . . . . . . . . 9

2.2 Different Access Technologies . . . . . . . . . . . . . . . 10

2.3 Access in Broadcast Networks . . . . . . . . . . . . . . . . . . 11

2.4 Distributed Coordinated Function Flowchart $[8] \ldots \ldots$. . . . . . . 12

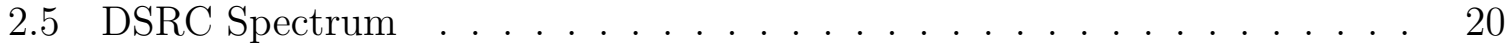

2.6 WAVE Protocol Stack[33] . . . . . . . . . . . . . . . . 22

$2.7 \quad$ IEEE802.11p/1609.4 Multichannel Operation . . . . . . . . . . . . . 22

2.8 VMESH with IEEE802.11p/1609.4 Multichannel Operation[35] . . . . . 23

3.1 ISM and UNII-3 Band directly before DSRC Band . . . . . . . . . . 31

3.2 Control Frames Used on Dedicated Control Channel . . . . . . . . . . . . 33

3.3 Channel Neighbor State Table (CNST) . . . . . . . . . . . . . . 35

3.4 Proposed Protocol's Modes of Operation . . . . . . . . . . . . . 37

3.5 Dynamic Channel Allocation Protocol Flowchart . . . . . . . . . . . . 38

4.1 MAC Issues in VANets . . . . . . . . . . . . . . . . . . 45

4.2802 .11 Different Transmit Operation Mechanisms . . . . . . . . . . . 46

4.3 Comparison between driving within and out of communication range . . 52

4.4 Calculating the Direction Angle . . . . . . . . . . . . . . 54

5.1 MQOG MAP for Simulation . . . . . . . . . . . . . . 60 
5.2 EMQOG MAP for Simulation . . . . . . . . . . . . . 61

5.3 Delay of Safety Message Reception . . . . . . . . . . . . . . . . . 65

5.4 Packet Delivery Ratio for Safety Messages . . . . . . . . . . . . . . 66

5.5 Throughput $x$ Number of Vehicles . . . . . . . . . . . . . . 68

5.6 Throughput $\mathrm{x}$ Traffic Rate . . . . . . . . . . . . . . . . . 68

5.7 L2 Retransmission Rate . . . . . . . . . . . . . . . . . . 69

5.8 Average Delay for Safety Message Reception - Scenario A . . . . . . . . . 72

5.9 Average Delay for Safety Message Reception - Scenario B . . . . . . . . . 72

5.10 Packet Delivery Ratio - Scenario A . . . . . . . . . . . . . . . . 74

5.11 Packet Delivery Ratio - Scenario B . . . . . . . . . . . . . . . 74

5.12 Average Throughput x Traffic Rate - Scenario A . . . . . . . . . . . 76

5.13 Average Throughput x Traffic Rate - Scenario B . . . . . . . . . . . 76 


\section{List of Acronyms}

AC: $\quad$ Access Category

ACK: Acknowledgement

AIFSN: $\quad$ Arbitrary Interframe Space Number

AP: $\quad$ Access Point

ASDM: $\quad$ Adaptive Space Division Multiplexing

BSS: $\quad$ Basic Service Set

BSSID: $\quad$ Basic Service Set Identification

CCA: Clear Channel Assessment

CCH: Control Channel

CCI: $\quad$ Co-Channel Interference

CDMA: $\quad$ Code Division Multiple Access

CNST: Channel Neighbor State Table

CSMA: $\quad$ Carrier Sense multiple Access

CSMA/CA: Carrier Sense multiple Access / Collision Avoidance

CSMA/CD: Carrier Sense Multiple Access / Collision Detection

CTP: Channel Transmit Power

CTS: $\quad$ Clear To Send

CW: Contention Window

DCA: $\quad$ Distributed Channel Access

DCF: $\quad$ Distributed Coordination Function

DIFS: $\quad$ Distributed Interframe Space

DIVA: Developing Next Generation Intelligent Vehicular Networks and Applications

DRP: Distributed Reservation Protocol

DRR: Data Rate Ratio

DSA: $\quad$ Dynamic Spectrum Access 


$\begin{array}{ll}\text { DSRC: } & \text { Dedicated Short Range Communication } \\ \text { EDCA: } & \text { Enhanced Distributed Channel Access } \\ \text { EMAP: } & \text { Electronics Maps } \\ \text { EMQOG: } & \text { Enhanced Multichannel QoS Cognitive MAC } \\ \text { ETSI: } & \text { European Telecommunications Standards Institute } \\ \text { FCC: } & \text { Federal Communications Commission } \\ \text { FCFS: } & \text { First Come First Serve } \\ \text { FDMA: } & \text { Frequency Division Multiple Access } \\ \text { GPS: } & \text { Global Positioning System } \\ \text { HCF: } & \text { Hybrid Coordination Function } \\ \text { IC: } & \text { Industry Canada } \\ \text { ISM: } & \text { Industrial, Scientific and Medical Band } \\ \text { LDMA: } & \text { Location Division Multiple Access } \\ \text { LLC: } & \text { Logic Link Control } \\ \text { LOP: } & \text { Link Out of Range Prediction } \\ \text { MAC: } & \text { Medium Access Control } \\ \text { MANets: } & \text { Mobile Ad Hoc Networks } \\ \text { MOBYTOP: } & \text { Mobility Based Dynamic Transmit Opportunity } \\ \text { MQOG: } & \text { Multichanel QoS Cognitive MAC } \\ \text { MSDU: } & \text { MAC Service Data Unit } \\ \text { NAV: } & \text { Network Allocation Vector } \\ \text { OCC: } & \text { Optical Orthogonal Codes } \\ \text { OFDM: } & \text { Orthogonal frequency-division multiplexing (OFDM) } \\ \text { OSI: } & \text { Open Systems Interconnection } \\ \text { PCF: } & \text { Point Coordination Function } \\ \text { PCS: } & \text { Physical Carrier Sense } \\ & \end{array}$


PDR: $\quad$ Packet Delivery Ratio

PHY: Physical

QOS: Quality of Service

R-ALOHA: Reservation Aloha

RF: Radio Frequency

RMS: $\quad$ Root Mean Square

RSS: Radio Signal Strength

RSSI: Radio Signal Strength Indicator

RTS: Request To Send

SCH: $\quad$ Service Channel

SDMA: $\quad$ Space Division Multiple Access

SIFS: $\quad$ Short Interframe Space

SSA: $\quad$ Static Spectrum Access

SSID: $\quad$ Service Set Identifier

SUMO: $\quad$ Simulation of Urban Mobility

TDMA: $\quad$ Time Division Multiple Access

TP: $\quad$ Transmit Power

TraCI: $\quad$ Traffic control Interface

TXOP: $\quad$ Transmit Opportunity

UNIII: Unlicensed National Information Infrastructure

VANETS: Vehicular Ad hoc Networks

VCS: Virtual Carrier Sense

WAVE: Wireless Access in Vehicular Environments

WBSS: $\quad$ Wave Basic Service Set

WLAN: Wireless Local Area Network

WMM: $\quad$ Wireless Multimedia 


\section{Chapter 1}

\section{Introduction}

Since the last few years, VANets (Vehicular Ad Hoc Networks) has been the focus of many automotive industries and academic research communities due to their distinct advantage and diverse applications. Research in this field has witnessed wide support from various governments and safety organizations since a well established vehicular network provides an efficient transportation system, safety for passengers, and onboard passenger's services as vehicles are becoming part of the next generation global internet of things.

Transportation Efficiency: According to [1], today there are approximately 6.8 billion people in the world and by 2044 that number will grow to about 9 billion. This would result in many problems one of which is the transportation system. As the total number of vehicles is growing from 800 million cars today to $2-4$ billion by 2050 , global gridlocks and traffic jams will occur in many different places. Not only people would be wasting their time stuck in traffic jams, but also the gridlock would stifle economic growth and the ability to deliver food and health care particularly to people that live in city centers. As we do not have space to build new roads, the solution will not definitely be building more transportation systems but integrate the current one to become smarter 
and more efficient. This would reduce congestion and consequently fuel consumption and enhance economic productivity.

Passengers' Safety: By the end of 2010, World Health Organization (WHO) estimated that nearly 3,500 people died on the world's roads every day and millions of people were injured or disabled every year[2]. Many accidents may be circumvented if the vehicles are able to communicate with each other to warn passengers while driving. Safe driving applications and services may include emergency vehicle warning (sudden breaking, icy road or oil stain, approaching vehicle, stop sign warning for reckless driving, highway-rail intersection warning and others)

Passengers' Onboard Applications and Services: Numerous applications and services included in the car would render driving more time-efficient, pleasant and entertaining. For example, applications such as searching directly for restaurants while calculating the shortest routes, internet access, downloading Emaps, sending emails, voice or video conversations with neighboring vehicles may all be some applications used onboard. Services might include finding parking spots and paying electronically. Toll services and payment, social networking and communicating with different service providers may be other services integrated in the network.

In general, VANet is going to be part of our system in the near future because of the vital needs and benefits it provides once established. What makes it more promising and pushing for its rapid development are the availability of low cost GPS and the drop in cost and wide adoption of 802.11 as a WLAN (wireless local area network)[3]. Moreover, local regulatory entities such as FCC (Federal Communications Commission in the US), ETSI (European Telecommunications Standards Institute in Europe) and IC (Industry Canada in Canada) have all laid the cornerstone for the initiation of vehicular networks by allocating a unique spectrum the DSRC (Dedicated Short Range Communications $5.9 \mathrm{GHz}$ ) for vehicular communication [4]. 


\subsection{Motivation}

One of the most challenging parts in the design of a vehicular network lies in the design of an efficient MAC layer that considers both the highly dynamic nature of vehicles and the different QoS (Quality of Service) applications thus supported. Many proposed MAC protocols for VANets consider one aspect but overlook others. We are in need for a MAC protocol that is capable of providing an integrated solution while dealing with different challenges simultaneously.

A MAC protocol for VANets should be designed for both urban and suburban environments. Since each environment has its own characteristics, the medium contention in congested environments (downtown of urban cities) is much more demanding than suburban ones. Therefore the MAC protocol should easily adapt to different traffic conditions.

Moreover, high mobility imposes high multipath environment with dynamic delay spread due to multiple reflections, scattering, diffraction and refraction. Therefore there exists a need in vehicular environments to assess the channel dynamically before initiating transmission as many cars are competing for a limited spectrum resource in a network that is suffering frequent connections and disconnections.

On the other hand, there exists an imposed latency requirement for QoS applications and services. Data transmitted over VANets can be classified as Safety or NonSafety with different considerations. QoS applications can be time-sensitive and latency-nontolerant and they range from Safety (collision warning), Realtime(video and audio) and NonRealtime (email, web surfing) applications. Safety messages must be sent within a certain upper threshold know as delay bound otherwise they reach their target late and are considered useless. En efficient medium access methodology ensures the prioritization of transmission by granting safety messages the highest priority to access the medium. 
Table 1.1: Examples of DSRC Applications and Requirements[5]

\begin{tabular}{lll} 
Application & Allowable Latency (ms) & Priority \\
\hline Intersection Collision Warning/Avoidance & 100 & Safety \\
Emergency Braking Warning / Avoidance & 100 & Safety \\
Cooperative Collision Warning & 100 & Safety \\
Toll Collection & 50 & Non-Safety \\
Service Announcements & 500 & Non-Safety \\
\hline
\end{tabular}

Table 1.1 shows different applications with different delay bound requirements[5].

Finally, a fair MAC protocol should consider the different vehicular speeds before and during the contention process. Thus a car moving with high speed should be able to contend faster and maintain its connection longer than a car moving with low speed.

According to above discussions, existing MAC protocols for VANets do not suit all of the above challenges. Some protocols tackle one challenge while overlooking others. Therefore, a new MAC protocol should be designed that is capable of prioritizing traffic to ensure QoS, mitigating interference in high multipath vehicular environment, considering high mobility and maximizing system throughput by introducing a unique multichannel cognitive operation.

\subsection{Thesis Objective}

The main objective of this thesis is to design and implement a MAC protocol for vehicular communication that can provide fault tolerance and fast response to topology changes, mitigate traffic congestion in urban scenarios, prioritize safety applications and services, and provide fair effective sharing of spectrum resources. Towards this objective, this thesis addresses the following relevant work: 
- A comprehensive study of current MAC protocols for VANet communications. The related work to be studied passes over the different MAC layer implementations. Those different architectures range from single to multichannel operation while using different channel access techniques. Pros and Cons of the different architectures will be presented in a table summarizing their features and comparing them to one another. Moreover, a detailed study of the IEEE802.11p will be presented which is the IEEE standard for the PHY and MAC layer ratified in 2010 for vehicular communication.

- An efficient Multichannel QoS Cognitive MAC (MQOG) will be proposed. MQOG will address the issues of granting safety messages a fast access to the spectrum, providing a fast response to any topological changes and mitigating interference in high multipath vehicular environment. Moreover, it will enhance throughput, minimize delays and ensure the reliable transmission of safety packets.

- An enhanced version of MQOG will be proposed (EMQOG). EMQOG will incorporate mobility, QoS, and download data rate on top of the multichannel operation. It will be able to provide fair effective sharing of radio resources among different vehicles. EMQOG will use a novel mobility based dynamic TXOP scheme (MoByToP) added to the MAC layer to extend the operation of the medium reservation phase.

- Performance evaluation will be performed for both MQOG and EMQOG. Both MAC protocols will be compared to IEEE802.11p and other existing protocols. Different scenarios will be used and the results will be all captured, compared and studied. 


\subsection{Contribution}

The main contributions of this thesis are:

1. An efficient multichannel QoS Cognitive MAC (MQOG ) for vehicular communication is designed and developed. This MAC protocol is accompanied with a new MAC architecture and operation deploying two new protocols: a channel allocation and a channel negotiation protocol. Moreover new control frames and protocol tables are presented to work along the proposed MQOG protocol.

2. A novel mobility based dynamic TXOP (MoByToP) is developed for the first time. MoByToP is added on top of MQOG to have an enhanced version of MQOG able to effectively share radio resources while incorporating mobility, QoS and download data rates.

\subsection{Thesis Organization}

The rest of the thesis is organized as follows:

- Chapter 2 presents a background study of already existing MAC protocols for VANets based on the different architectures used and compares the distinct features of those protocols with one another.

- Chapter 3 gives an overview of the proposed Multichannel QoS Cognitive MAC (MQOG) followed by a detailed explanation of the proposed protocol by describing its architecture, the dynamic channel allocation and channel negotiation protocols involved.

- Chapter 4 gives an overview of the mobility based dynamic TXOP (MoByToP) for VANets followed by a detailed explanation of the protocol by describing its 
constituent parts the QoS, mobility and transmission data rate involved. Integrating MoByToP with MQOG is then explained to result with Enhanced MQOG (EMQOG).

- Chapter 5 describes in details the performance evaluation methodology and results thus obtained. The simulation software, mobility model, and the different scenarios are thoroughly explained along with the different metrics used to evaluate MQOG and EMQOG. Comparison of results is also presented while comparing them to already existing MAC protocols.

- Chapter 6 gives concluding remarks of our work and outlines the possible future work. 


\section{Chapter 2}

\section{Related Work}

In this chapter, we first explain the medium access control's role in the VANet environment then we highlight design guidelines needed for the appropriate design of an efficient MAC layer. In addition, we will discuss existing related work on VANet's MAC covering different architectures by identifying each architecture pros and cons.

\subsection{Background}

\subsubsection{MAC: Medium Access Control}

As described in the OSI model, the MAC sublayer is a subset of the data link layer shown in Figure 2.1. The MAC sub-layer acts as an interface between the logical link control (LLC) sublayer and the network's physical layer. The MAC provides addressing and channel access control mechanisms to coordinate the transmission between users sharing the medium. The MAC sublayer makes it possible for several nodes to communicate within a multiple access network that incorporates a shared medium[6].

As shown in Figure 2.2, the different MAC protocols can be classified as channel or packet based. This is due to the fact that there exists two types of networks: Switched 


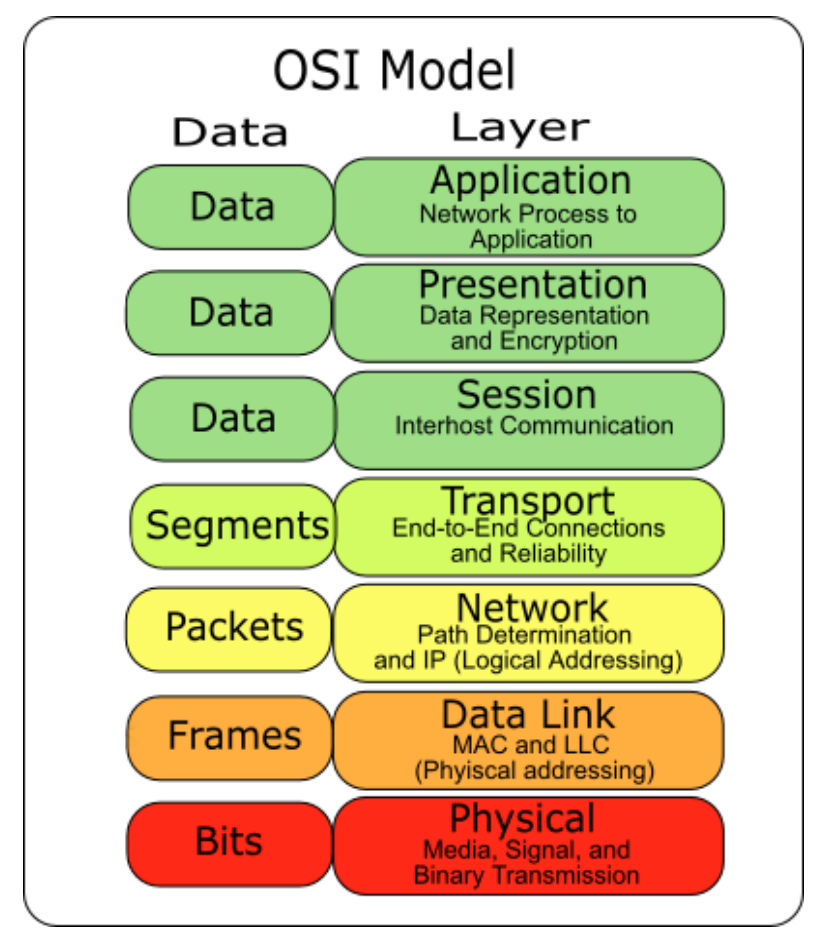

Figure 2.1: OSI Model

and Broadcast networks. For switched networks (channel based approach) a dedicated line of connection is formed between the source and the destination. Different channel access techniques can be implemented such as Frequency Division Multiple Access (FDMA) where different users have different frequencies; Time Division Multiple Access (TDMA) where users access within different time slots; Code Division Multiple Access (CDMA) where users acquire different codes and Space Division Multiple Access (SDMA) where users access the medium according to their specific location. Other channel based multiple access techniques can be used by having different combinations between FDMA, TDMA, CDMA and SDMA. For example GSM a 2G cellular technology uses FDMA and TDMA. Switched networks usually provide better quality than broadcast networks (packet based approach) because of the dedicated connection however this is a consequence of higher costs[7]. 


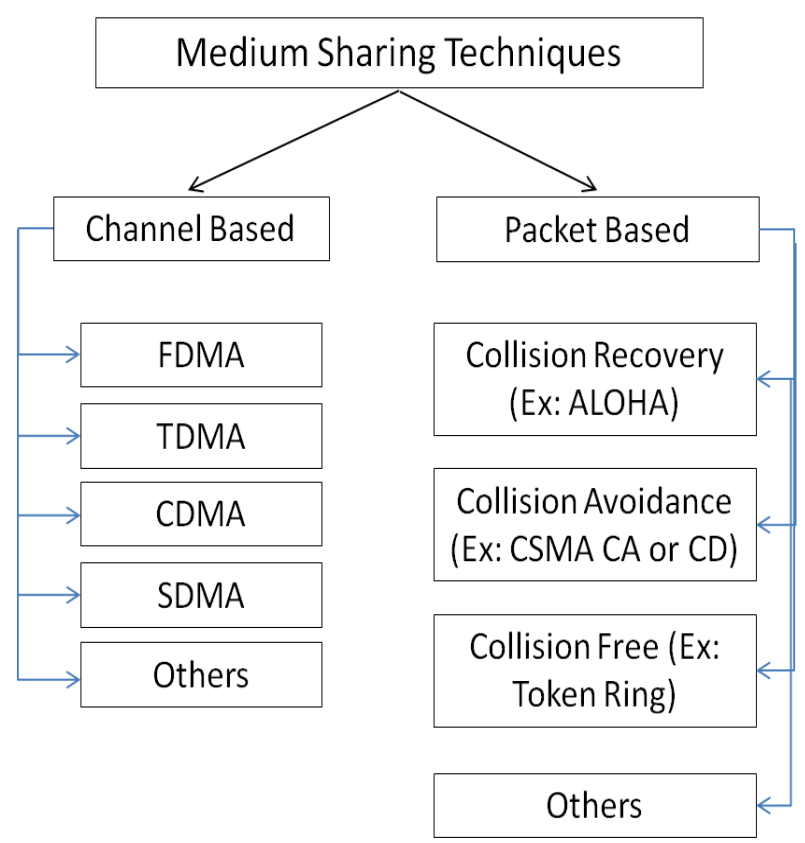

Figure 2.2: Different Access Technologies

On the other hand, broadcast networks provide a single shared medium where messages are received by all stations. For broadcast networks, any transmission from any station can be heard by any other stations. If two or more stations transmit at the same time then collision occurs. Vehicular environments are considered broadcast networks as different vehicles contend for the same shared medium. As depicted in Figure 2.3, many nodes try to access the medium and it is the role of the MAC layer to coordinate their access. Packet Based MAC approach can be classified as collision recovery such as ALOHA where many vehicles transmit and wait for an acknowledgment. Unless the acknowledgment is received, the vehicle has to transmit the packet again. Collision avoidance approaches are the IEEE802.2 Ethernet (Collision detection) and the IEEE802.11 WLAN (Collision Avoidance). The collision detection bake offs transmission when detecting a collision and the collision avoidance ensures the medium is empty for transmission prior to transmission. Collision free approaches are the scheduled based where vehicles access the medium whenever they have the token. It is similar to the channel based TDMA 
approach where vehicles access the medium one after the other in a round robin fashion. Other packet based approach can also be considered and different combinations can be set up.

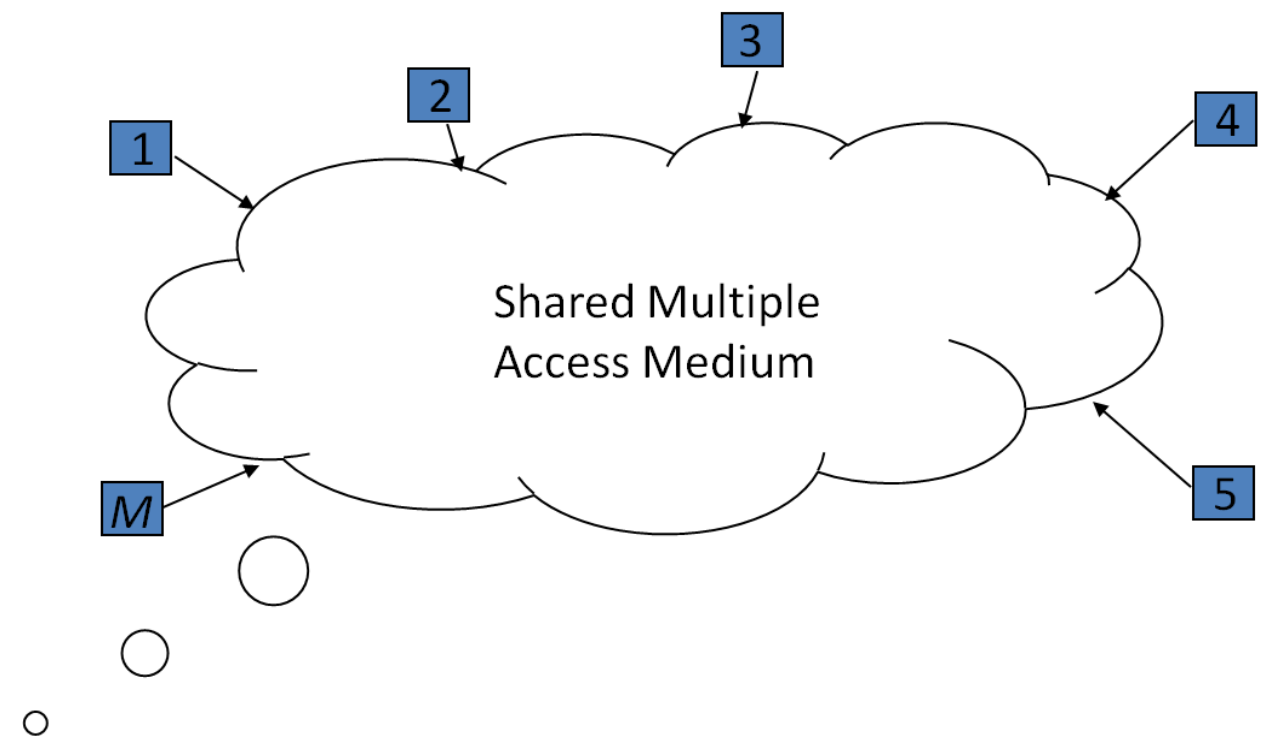

Figure 2.3: Access in Broadcast Networks

\subsubsection{IEEE 802.11 MAC Distributed Coordination Function $(\mathrm{DCF})$}

In this section, I explain the detailed distributed coordination function DCF of IEEE802.11 [8]. In IEEE 802.11, there are two methods to access the medium. Distributed Coordination Function (DCF), based on CSMA/CA, used to coordinate the medium access in the ad hoc mode. The other function is point coordination function (PCF) which is used to control the medium access in a centralized mode. The majority of the protocols proposed for vehicular networks require operating in an ad hoc mode. I start by explaining DCF as in my proposed protocol I modify some parts of its operation. DCF is the mandatory access method of the 802.11 standard while Point Coordination Function 
(PCF) is optional. The DCF is mainly used in all wireless LANs networks nowadays. Figure 2.4 is a flowchart that explains the detailed operation of DCF $[8][9]$.

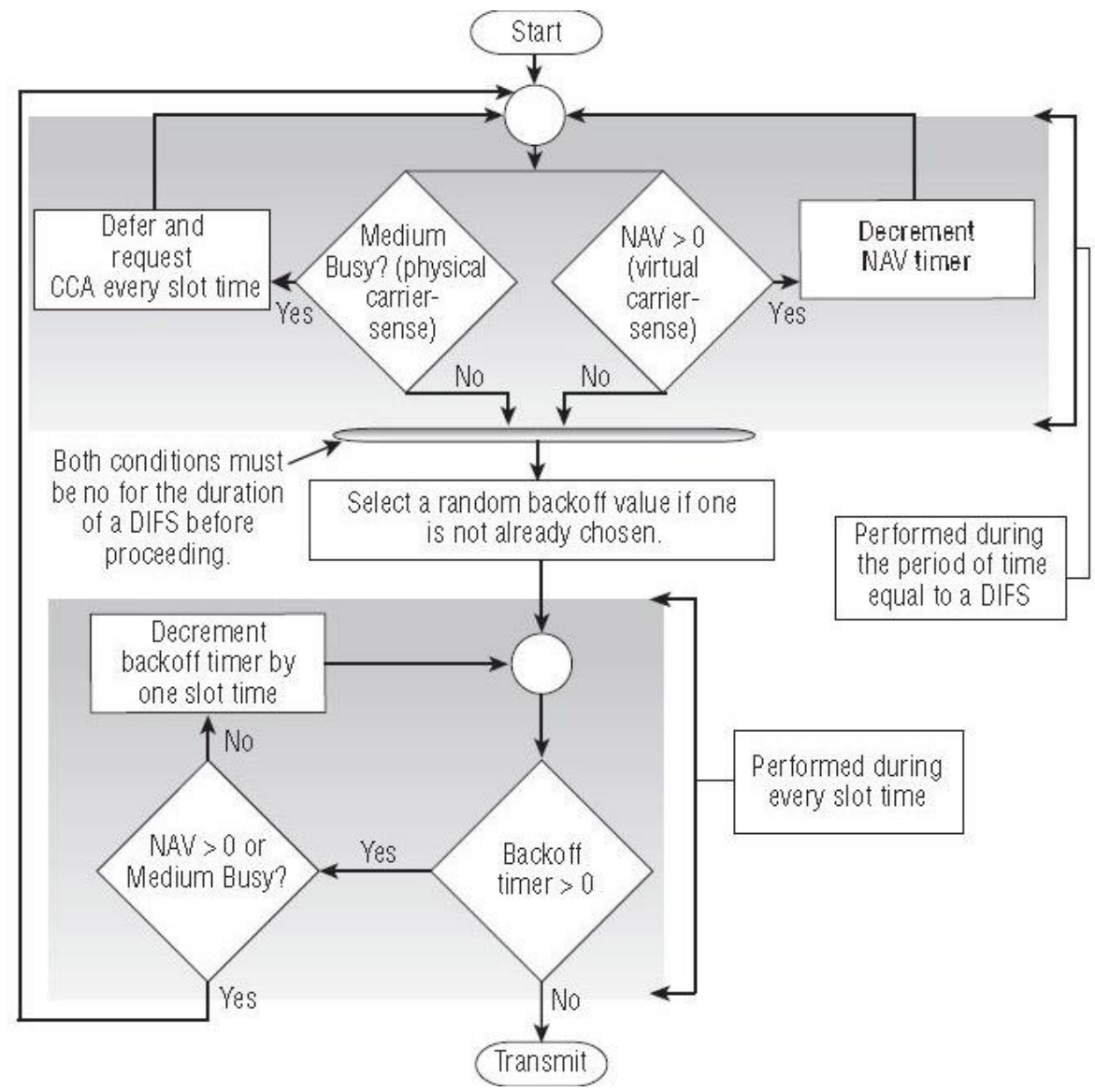

Figure 2.4: Distributed Coordinated Function Flowchart[8]

1. Before a node attempts to send a packet both the physical carrier-sense and virtual carrier-sense should be tested. The physical carrier sense determines whether the medium is busy before transmission by listening to the signal strength at the physical layer. We call this process clear channel assessment. The virtual carrier sense uses NAV timer (network allocation vector) to maintain a prediction of future traffic on the medium based on the duration field. If the NAV is greater than 0 , this means that another station is transmitting and the station has to wait. The NAV is 
then decremented, and the flowchart loops back toward the beginning. If the clear channel assessment (CCA) indicates that the medium is busy, this also means that another station is transmitting and the station has to wait.Then the station must wait a slot time and loop back toward the beginning to perform another CCA.

2. When both PCS and VCS do not detect any wireless traffic for a period of a DIFS, the station selects a random backoff value. The backoff timer decision point is then tested every slot time to see if the backoff value is greater than 0 . If it is greater than 0 , the station needs to test again for the PCS and VCS.

3. If the medium is idle, the station will decrement the backoff timer, and then go back to the backoff timer decision point to see if the timer has reached 0 yet. When it reaches 0 , the station can transmit one frame.

\subsection{Medium Access Control Design Guidelines}

The MAC designed for a VANet should be able to provide the following:

1. Provide Fair/Effective sharing of radio resources: In general, at the MAC level users should be able to transmit with equal probability of transmission. However in vehicular networks, fairness has a different approach. Due to high mobility, different QoS requirements and different download data rates, vehicles should be able to effectively share radio resources based on particular dynamic conditions. For example, a car moving with high speed should have faster access to the medium than a car moving with low speed as its time for medium contention is much less than the slow car.

2. Minimize L2 Retransmissions by Preventing or Reducing Packet Collisions : Any efficient MAC protocol should prevent packet collisions and consequently reduce 
frame retransmissions. Layer 2 retransmissions are the mortal enemy of any MAC protocol[9]. As all MAC frames should be acknowledged, failing to receive an ACK will result in a retransmission. Excessive L2 retransmissions affect the network in 2 ways: First they increase overhead and therefore decrease throughput. Secondly, increase delay and jitter problems for time-sensitive applications such as voice and video and hence degrade the performance of the network. Most data applications can handle L2 retransmissions up to $10 \%$ but time sensitive application should have an L2 retransmission less than $5 \%$.

- Minimize Packet Delays: An important requirement for vehicular communications is that a message should be delivered within a certain time. This time is known as communication delay bound, and can be defined as the maximum time duration between the generation and the successful reception of that message.

- Maximize Throughput: Efficient MAC protocols tend to maximize throughput when they reduce or prevent packet collisions. Any packet collision would result in decrease in throughput.

- Maximize Packet Delivery Ratio (PDR): PDR is a measure of the transmission reliability of the MAC protocol. PDR requirement depends on the type of application. The PDR should be larger than a certain threshold to provide a specific service. For safety applications PDR should be above 90 $\%$ [8]. To achieve a desired PDR, two factors can be dealt with at the MAC level: collisions and transmission interference.

3. Co design MAC and QoS by prioritizing Safety packets over nonSafety Packets : In computer networks, we do not face critical safety applications. The differentiation among packets is between realtime and nonrealtime applications where realtime 
applications and services require faster access and hence different protocols than nonrealtime applications. An efficient MAC for VANets should be able to prioritize safety packets over nonsafe ones. Access for safety messages should be faster and more reliable than nonsafe messages.

In the following section, I will start my discussion by highlighting the single radio single channel architecture solutions [10-29] then move to the single radio multichannel solutions [30-35] and finally to the multiradio multichannel solutions [36-37].

\subsection{Single Radio Single Channel Solutions}

The different MAC protocols for Vehicular Ad Hoc Networks were widely investigated in the literature [10-37]. MAC protocols vary depending on the different MAC architecture used. I will start my discussion with single radio single channel solutions. The following are divided into space division multiple access, repetition based MAC, Adhoc MAC and directional antenna based MAC.

\subsubsection{Space Division Multiple Access (SDMA) Solutions}

SDMA assigns channel access based on location information. Geographical areas are divided into smaller divisions based on real time position information. Each spatial region is allocated a unique channel and vehicles obtain information about channels using GPS coordinates. SDMA could work with multiple access schemes like TDMA, CDMA and FDMA for bandwidth sharing. SDMA aims to reduce access collisions, increase channel reusability and address issues likes hidden-node and fairness.

Applications of SDMA for VANets were proposed in [10],[11]. In [12], authors presented the Location Division Multiple Access (LDMA) protocol. LDMA assigns grid resolution and divides a geographical map into a hierarchy of spatial regions where cell 
sizes are based on geographic locations (urban, suburban and rural). For example, geographic locations with higher traffic density are assigned a higher grid resolution with a smaller cell size. LDMA uses GPS for location information and proposes the use of low cost, low rate FM-based Radio Data Broadcast System (RDBS) for distribution of spatial slot distribution and temporal schedule assignments. In case where there are multiple vehicles in the same cell, LDMA ensures that only one vehicle forwards the message by implementing small jitter duration (500 us) in the beginning of each time slot. Authors demonstrate that LDMA offers smallest end-to-end delay with moderate message receive rates when they compare it with other rebroadcast schemes.

As LDMA suffer from bandwidth wastage in case of empty cells, the concept of buffer was presented in [13]. Authors in [13] developed Adaptive Space Division Multiplexing (ASDM) technique that allows vehicles to transmit using time slot of empty cells as they adopt time slot assignments and lead vehicles up to the limit defined by ASDM buffer.

Another technique that uses SDMA with CDMA was proposed in [14]. The authors proposed a position based PN code assignment scheme to alleviate the problem of PN code assignment. Their scheme exploits the location awareness for efficient PN code allocation. Roads are divided into small segments or areas and a small number of PN codes are allocated to each segment or area. According to the scheme, vehicles use a multi MCS scheme to sense availability of PN code. The main advantage of this scheme is avoidance of PN code conflict as selection of code is based on location information.

SDMA has emerged as very promising technique. However, there are still practical challenges allied with SDMA technique, which necessitate further research. Main Issues involved in SDMA based MAC design are cell size, channel reuse-distance, accuracy of position acquisition systems, redistribution of schedule and division border effect [11][15]. 


\subsubsection{ADHOC MAC}

ADHOCMAC [16] is a MAC protocol that was proposed to achieve a distributed TDMA function without any centralized coordinator. It was developed under the CarTalk2000 project to support inter-vehicular communications[17]. Mainly, ADHOCMAC is based on Reliable R-ALOHA (RR-ALOHA) which is a MAC scheme capable of coordinating access in a distributed mode. The main advantage of ADHOCMAC is that it can be adapted to work with 802.11 physical layer by modifying its frame structure. As ADHOCMAC uses a dynamic TDMA mechanism, independent from the physical layer, it can coordinate access of ad hoc nodes by allocating dynamic time slots for the next packet transmissions. The protocol is supposed to mainly solve the hidden and exposed node problems and provide a reliable single hop broadcast service.

In [18][19], several issues regarding ADHOC MAC are discussed. First, in a static scenario the minimum time needed to successfully obtain the basic channel is greater than $200 \mathrm{~ms}$. Secondly, mobility and dense traffic may cause more latency in allocating and releasing slots. Thirdly, the number of vehicles in the same communication range must not exceed the number of slots otherwise there will not be enough slots for vehicles to transmit. In comparison with the IEEE 802.11, ADHOC MAC is not utilizing the medium efficiently. Further information about ADHOC MAC performance can be found in $[20]$.

\subsubsection{Repetition-based MAC}

The idea of repetition-based MAC is proposed in [21][22]. The aim of any repetitionbased MAC protocol is to deliver safety messages with high reliability and low delay by repeating the transmission and hence having a better probability of reception. On the other hand, undesirable amount of repetition may cause severe consequences. The repetition- based solution is to allocate a finite number of slots for repetition while the 
lifetime of a message is divided into several slots based on its useful lifetime and the transmission time. A MAC extension layer is added between the logical link layer and the MAC layer to handle the generation and removal of repetitions. The main advantage of this design is its simplicity.

In [23], authors utilize optical orthogonal codes (OCC) with the repetition process to minimize message loss probability, reach better probability of detection, and reduce the reception delay. The codes would help in finding the number of repetitions that would cause a collision and alleviate the corresponding packets. A similar approach was used in [24] to provide different QoS priority levels. In [25] a distributed feedback mechanism is used to optimize the number of repetitions where it broadcasts information regarding the transmission and reception of messages through the network. It uses index coding to minimize the number of transmissions.

In general, repetition based solutions may provide better probability of reception however they all require MAC extensions to handle the generation and removal of repetitions.

\subsubsection{Directional Antenna-Based MAC}

As vehicles in VANets move according to road geometry, the transmission should be done in specific directions. For example, in the sudden breaking safety application only vehicles behind the car should be warned while vehicles in front are not affected. Instead of using omni-directional antennas, directional antennas would not only circumvent unnecessary transmission but also overcome problems such as interference, hidden node and exposed node problems. Moreover, increased transmission range, and reuse of channels are the main benefits brought by those protocols.

In [26][27][28], several MAC protocols adopt directional antennas and achieve network

performance improvement. However, the main issues facing directional antennas are the 
complexity and difficulties of providing practical implementation in VANets. For more information about MAC protocols with directional antennas, [29] is considered a good reference that provides a classification of MAC protocols with directional antennas, and discusses the challenges in their design.

Since the nature of vehicular communication imposes a distributed architecture where there isn't any centralized controller managing transmissions, multichannel solutions were shown to be more suitable for VANets than single channel protocols [30-37]. This is due to the fact that multichannel solutions ensure intelligent control and coordination between vehicles on a particular channel.

\subsection{Single Radio Multichannel Solutions}

Multichannel solutions can be divided into 2 parts: Single radio Multichannel[30-35] and multiradio multichannel[36-37]. I will begin my discussion with single radio multichannel then move to multiradio multichannel.

\subsubsection{IEEE802.11p Protocol}

IEEE ratified 802.11p in 2010 as WAVE Amendment (Wireless Access in Vehicular Environments) defining the PHY and MAC layer for VANets operating in 5.9 GHz Band[30]. IEEE 802.11p divided the $75 \mathrm{MHz}$ DSRC Band into seven $10 \mathrm{MHz}$ channels as shown in Figure 2.5, composed of one control channel $(\mathrm{CCH})$ which is assigned for broadcast, safety and control messaging and six service channels (SCHs) for ongoing data transactions.

IEEE 802.11p WAVE MAC Amendments: In general, the IEEE 802.11 MAC operations are time consuming to be adopted by IEEE 802.11p. Vehicular safety ap- 


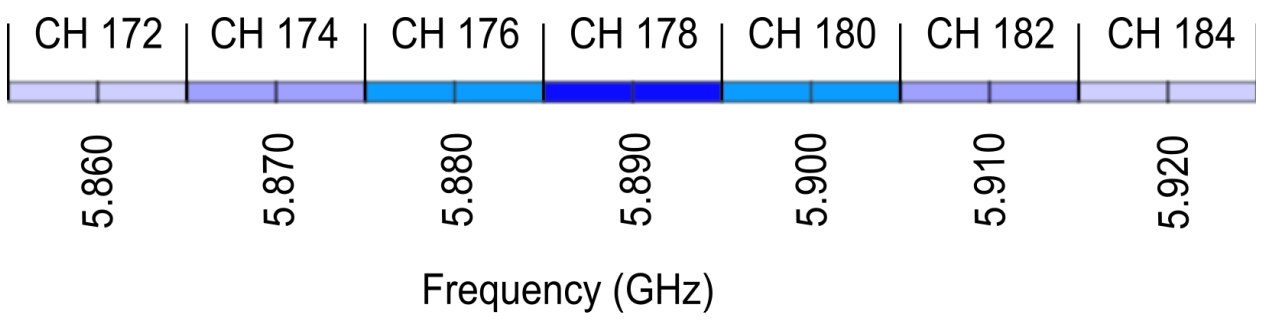

Figure 2.5: DSRC Spectrum

plications require instantaneous data exchange and cannot afford scanning channels for the Access Point (AP) beacon of a Basic Service Set (BSS) and subsequently executing multiple handshakes to establish association and dissociation. Therefore, it is essential for all IEEE 802.11p radios to be in the same channel and configured with the same BSSID to enable safety communications. A key amendment introduced by the IEEE 802.11p WAVE is the term "WAVE mode"[30]. A WAVE BSS (WBSS) is a type of BSS consisting of a set of cooperating stations in WAVE mode that communicate using a common BSSID. When a radio in WAVE mode sends a WAVE beacon including all necessary information for a receiver to join, a WBSS is initialized. A radio joins a WBSS when it is configured to send and receive data frames with the BSSID defined for that WBSS. So a station in WAVE mode is allowed to transmit and receive data frames with the wildcard BSSID value i.e. two vehicles can immediately communicate with each other upon encounter without any additional overhead[33]. In addition, IEEE802.11p uses the Enhanced Distributed Channel Access (EDCA) mechanism to ensure QoS prioritization[31]. 802.11e is also known as WMM (wireless multimedia). It defines 4 access categories (Voice, Video, Best effort and background) for traffic prioritization. Those access categories are mainly used to prioritize real time over non realtime applications however they do not differentiate safety from non safety applications and thus considered insufficient for vehicular communications. 
IEEE 802.11p WAVE PHY Amendments: At PHY level, IEEE 802.11p is designed to make the minimum necessary changes to IEEE 802.11 PHY. This approach is feasible because IEEE 802.11a radios already operate at $5 \mathrm{GHz}$ and it is not difficult to configure radios to operate in the $5.9 \mathrm{GHz}$ band in the U.S. and similar bands internationally. IEEE 802.11p is essentially based on the OFDM PHY defined for IEEE 802.11a, with a $10 \mathrm{MHz}$ wide channel instead of the $20 \mathrm{MHz}$ one usually used by $802.11 \mathrm{a}$ devices[33]. The key reason is to address the increased RMS delay spread in the vehicular environments. Guard interval at $20 \mathrm{MHz}$ is not long enough to offset the worst case RMS delay spread (i.e. to prevent inter-symbol interferences within one radio's own transmissions in the vehicular environments). IEEE 802.11p PHY amendment also improves the receiver performance requirements and transmission mask.

As shown in Figure 2.6, IEEE 802.11p WAVE is only a part of a group of standards related to all layers of protocols for DSRC based operations[33]. Just like IEEE 802.11, IEEE 802.11p defines the MAC and PHY layers of the WAVE protocol stack. The upper level layers are defined in the IEEE 1609 [34] family of standards. IEEE 1609 is divided into four standards:

1. IEEE P1609.1: Resource Manager

2. IEEE P1609.2: Security Services for Applications and Management Messages

3. IEEE P1609.3: Networking Services, WAVE connection setup and management.

4. IEEE P1609.4: Multi-Channel Operations.

IEEE1609.4 defines the multichannel operation of 802.11p where all devices participating should monitor the $\mathrm{CCH}$ where high priority control and safety messages are transmitted in that interval. Figure 2.7 shows the 1609.4 multichannel operation with 


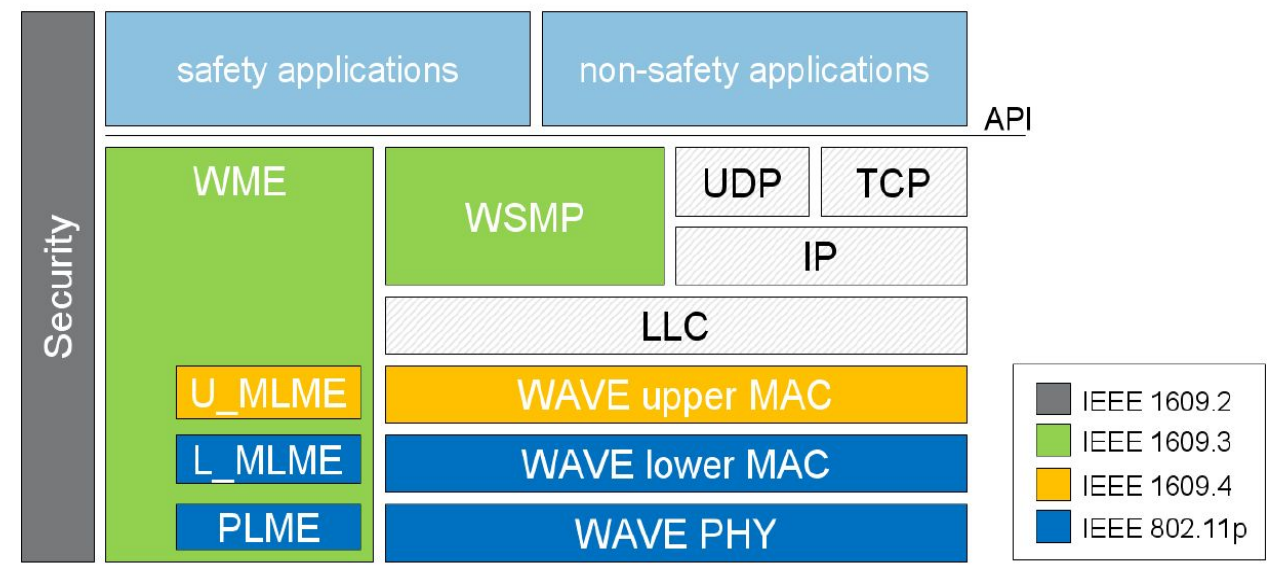

Figure 2.6: WAVE Protocol Stack[33]

a sync interval for time synchronization between vehicles within the same transmission range. For more information on IEEE 1609.4 refer to [34].

I Guard Interval

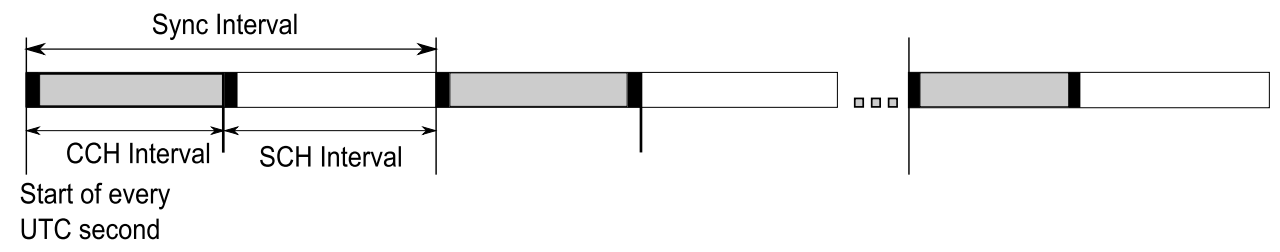

Figure 2.7: IEEE802.11p/1609.4 Multichannel Operation

The main issues faced when using 802.11p/IEEE1609.4 are the overhead encountered on the control channel because of control, safety and broadcasting messages and hence the delay on the medium contention increases. In addition, time synchronization in multichannel operation between vehicles is hard to achieve in high multipath environments. 


\subsubsection{VMESH: Distributed Segment Storage for Peer-to-Peer Streaming}

In [35], authors propose VMESH, an extension to IEEE 802.11p with Multichannel operation . VMESH uses a contention-based access method for control channel and a contention-free (TDMA) access method for service channels in parallel with 802.11p multichannel operation. As presented in Figure 2.8, VMESH encompasses a superframe on top of wave synchronization interval, which contains multiple synchronization intervals. Control channel interval is divided into Beacon Period (BP) and Safety Period (SP). During the Beacon Period, stations contend for medium access for beacon slots using ALOHA. The beaconing scheme uses a Distributed Reservation Protocol (DRP) to ensure contention free medium access on service channels using TDMA (time division multiple access). This reserves resources dynamically on service channels to improve performance of sensitive applications.

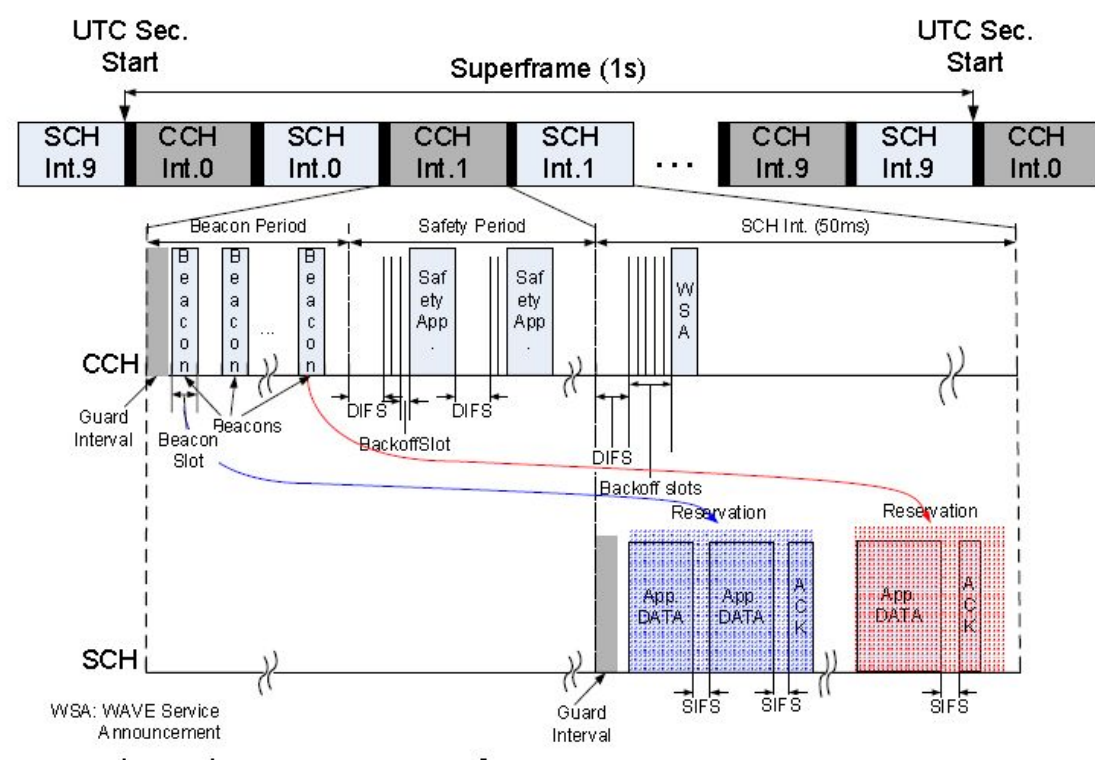

Figure 2.8: VMESH with IEEE802.11p/1609.4 Multichannel Operation[35] 
The downside of VMESH is that it doesn't ensure QoS neither between safety and nonsafety messaging nor between real time and non real time messaging. Moreover, using TDMA would limit the number of vehicles to the number of available slots and slots are wasted if no vehicles are using them on the service channel interval. Finally time synchronization is also hard to maintain as vehicular environments impose a high multipath and dynamic nature.

\subsection{Multiradio Multichannel Solutions}

The main advantage in using a multichannel multiradio solution is that while two radios are on simultaneously, the intelligence of a network increases. For example, one radio can scan for topological changes while the other one can transmit. Moreover, cluster based solutions require one radio to communicate with the clusterhead while the other to communicate with neighboring clusters. This comes as tradeoff between cost and efficiency as each vehicle would be equipped with 2 antennas with 2 radios working simultaneously. Multiradio Multichannel solutions discussed in this paper are clusterbased and dynamic channel assignment[36][37].

\subsubsection{Cluster Based MAC Solutions}

In [36] Su and Zhang propose a clustering-based multichannel MAC that uses contentionfree and contention-based MAC protocols. Platoons are cars moving along the same road with similar speed and therefore share similar properties. Those cars can be grouped together in a cluster and by using an election protocol, a cluster head is elected while others are considered cluster members. Su and Zhang define the use of TDMA for cluster members while cluster heads use 802.11p CSMA/CA to contend for the medium on a different frequency. Each vehicle has two transceivers operating simultaneously. The protocol is 
designed to provide QoS for realtime data, e.g. safety messaging, and it provides an increased throughput for non-realtime data.

The disadvantage of this protocol lies in the overhead due to the election process. Moreover two clusters operating on the same frequency create CCI (Co-Channel Interference) leading to degradation in performance. Synchronization is also an issue in this protocol when using TDMA in high multipath environments.

\subsubsection{On-Demand Channel Assignment (DCA) for Multichan- nel MAC}

In [37], the authors defined DCA for MANets (mobile ad hoc networks) based on dynamic channel assignment. DCA utilizes two transceivers; one always operates on a dedicated control channel while the other can be switched to any data channels in an on-demand manner. transmitter selects an empty frequency and sends it in the RequestTo-Send (RTS) packet to reserve the data channel and frequency for data transmission over the control channel. Upon receiving the RTS, the receiver decides on a channel and adds this channel reservation information to the CTS. Then, data and ACK packets are transmitted over the service data channel. In general, this protocol would select the least interfering frequency channel.

The downside of DCA is that below a saturation point (dependent on number of available channels) it can offer more throughput than others however when it reaches the saturation point, performance degrades. Moreover, as it is not designed for VANets, it doesn't consider QoS and mobility. Modification must be made to ensure a tailored version of this protocol for VANets. 


\subsection{Comparison of Existing Protocols}

We conclude the related work section by outlining the differences between the different VANet MAC protocols. To compare MAC protocols, certain criteria must be considered. Although in this case, we are comparing different MAC protocols qualitatively, the issues each protocol solved are emphasized, and the limitations are pointed out and they are all presented in Table 2.1.

Table 2.1: Comparison of Different MAC Protocols

\begin{tabular}{|c|c|c|c|c|c|c|c|}
\hline & Proposed & IEEE $802.11 p$ & ADHOC & VMESH & LDMA & Repetition & Cluster \\
\hline \multirow[t]{2}{*}{ Multiple Access } & CSMA & CSMA & TDMA & TDMA / & SDMA/ & ALOHA & CSMA/ \\
\hline & & & & CSMA & TDMA & & TDMA \\
\hline Reduce Collisions & YES & YES & YES & YES & YES & $\mathrm{NO}$ & YES \\
\hline Increase Channel & YES & YES & YES & YES & YES & YES & YES \\
\hline \multicolumn{8}{|l|}{ Reusability } \\
\hline Scalibility & YES & YES & $\mathrm{NO}$ & NO & $\mathrm{NO}$ & YES & YES \\
\hline Time & $\mathrm{NO}$ & - & YES & YES & YES & $\mathrm{NO}$ & YES \\
\hline Synchronization & & $(1609.4)$ & & & & & \\
\hline Use Backoff & - & YES & $\mathrm{NO}$ & - & $\mathrm{NO}$ & $\mathrm{NO}$ & $\mathrm{NO}$ \\
\hline \multicolumn{8}{|l|}{ Mechanism } \\
\hline Handles Hidden & YES & YES & YES & YES & YES & $\mathrm{NO}$ & YES \\
\hline \multicolumn{8}{|l|}{ Node } \\
\hline Ensure QoS & YES & - & NO & $\mathrm{NO}$ & $\mathrm{NO}$ & $\mathrm{NO}$ & - \\
\hline Fairness & YES & - & $\mathrm{NO}$ & NO & $\mathrm{NO}$ & $\mathrm{NO}$ & - \\
\hline Fault Tolerance & YES & - & NO & NO & $\mathrm{NO}$ & $\mathrm{NO}$ & $\mathrm{NO}$ \\
\hline
\end{tabular}


Until now there does not exist any complete solution suitable for all situations, scenarios, and QoS requirements. Although the IEEE802.11p is the most promising due to the popularity of 802.11 WLAN however it lacks some major requirements. This is what drove me to come up with a new multichannel cognitive MAC solution (MQOG) solely dedicated for vehicular communication.

\subsection{Summary}

In this chapter, I first presented related background followed by a literature review for MAC protocols in vehicular networks. MAC design guidelines were highlighted in the first part. After that, an overview of the existing MAC solutions for a vehicular environment was briefly introduced from the perspective of using different MAC architectures. This provided a broad view of the current existing MAC protocols for VANets. Finally, a qualitative comparison of existing protocols is provided. 


\section{Chapter 3}

\section{Proposed Multichannel QoS \\ Cognitive MAC for VANets \\ (MQOG)}

In this chapter I present our proposed Cognitive Multichannel MAC (MQOG) in detail. I first outline the motivation behind our work and then I go through the system architecture. Finally I explain the dynamic channel allocation and negotiation protocols used in my design.

\subsection{Motivation}

After examining the different related work, I find the following issues that should be considered in the efficient design of a MAC protocol for vehicular communications.

1. The MAC protocol designed should be able to prioritize safety messages on behalf of nonsafe messages. Moreover, not only should it prioritize safe messaging but also ensure their reliable transmission in case of facing contention on the medium. A 
dynamic solution should be available in case the medium is congested. Otherwise life-critical messages will miss their target.

2. As vehicular networks are of ad hoc nature then each vehicle should have enough knowledge and intelligence of its surroundings. The MAC protocol employed should be fault tolerant with respect to any topology changes. Any topological change should be accounted for directly. For example, if a vehicle is relying on an intermediate node to deliver a packet to the final destination and this node moves out of the network then it should be able to get notified directly.

3. As vehicular networks struggle from congestion in urban environments, it would result in an unstable, time-varying wireless channel. The MAC protocol designed should be able to assess the channel prior to transmission. Channel assessment for interference (Adjacent and Co-Channel Interference) would alleviate L2 packet retransmissions in high multipath environments with dynamic delay spreads.

Those needs urged us to design a cognitive Multichannel MAC for VANets able to address those issues as well as to achieve better MAC performance with respect to existing VANet MAC protocols.

\subsection{Cognitive Radios and Unlicensed Bands: Ap- proach}

There is a common belief that we are running out of usable radio frequencies because of the different wireless technologies being used nowadays. However actual spectrum usage measurements obtained by the FCC's Spectrum Policy Task Force shows that any given

time and location, much of the prized spectrum lies idle[38]. So there exists a need to efficiently manage the frequency spectrum as it is a limited resource that is not scaling 
with the increasing demands of wireless technologies. In general, we have two types of spectrum access: dynamic spectrum access and static spectrum access[38].

- Static Spectrum Access (SSA) has been used for long time in most wireless technologies. In static spectrum access transmissions based on applications are assigned static frequencies.

- Dynamic Spectrum Access (DSA) is a recent access technique where frequency selection is done on the fly due to different parameters. Advancements in antenna technologies had made this spectrum access feasible by introducing Cognitive Radios.

Cognitive radio, built on a software radio platform, is a context-aware intelligent radio potentially capable of autonomous reconfiguration by learning from and adapting to the communication environment [39]. While dynamic spectrum access is certainly an important application of cognitive radio, cognitive radio represents a much broader paradigm where many aspects of communication systems can be improved via cognition. Deploying cognitive radio in vehicular networks allows different channel selection dynamically while vehicles displace between different vehicular scenarios[39].

As already mentioned before, the FCC has allocated in 1999 the DSRC band for vehicular communications. As shown in Figure 3.1, directly before the DSRC band there exist the ISM and UNII-3 bands. Those bands are both unlicensed and free to use by any user except that the user must abide by the Channel Transmit Power (CTP) and Bandwidth Requirements set by the local regulatory entity in his country (FCC in the United States ; IC in Canada ; ETSI in Europe). In general, the $5.8 \mathrm{GHz}$ ISM and UNII3 are both used for outdoor communications with transmit power suitable for vehicular communications. Since those bands are free for use, the downside of using them is sharing the frequencies with too many other users (WLAN or other wireless technologies) 
leading to high level of interference.

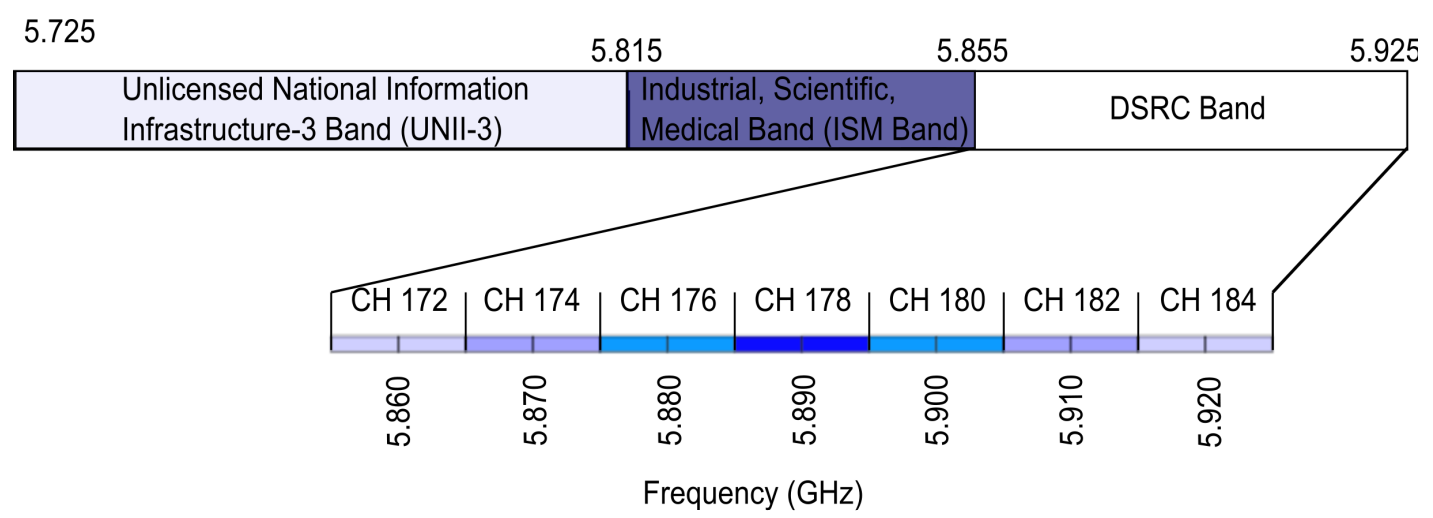

Figure 3.1: ISM and UNII-3 Band directly before DSRC Band

In my proposed protocol, I will use cognitive radios to transmit nonsafe data bursts in case all DSRC channels are occupied while we assess the channel before transmitting. Moreover, non safe data will handoff to an ISM or UNII-3 band in case a safety message has to be sent and the channel assessment didn't find any suitable channel on the DSRC band. Details of the proposed protocol are presented in the next sections.

\subsection{Proposed System Architecture}

MQOG uses multichannel operation with a unique dedicated control channel and multiple service channels for data transfer. The Dynamic Channel Allocation Protocol (refer to 3.4) ensures that each transmitter searches for the best available channel by assessing the noise and interference level on the DSRC, ISM, and UNII-3 bands. This protocol also considers the QoS of messages to be sent as it prioritizes frames based on safety or nonsafety applications.

In our proposed protocol, we separate the control from the actual data transmission. 
The underlying communication and intelligence lies on the control channel to dedicate a service channel for data transfer. All vehicles track the communication between neighboring vehicles using the Channel Neighbor State Table (CNST) (refer to 3.3.2). This table shows the transmissions of all neighboring vehicles in the range and hence a car while monitoring the control channel has enough intelligence to be aware of all transmissions occurring next to it.

The second protocol is the Channel Negotiation Protocol (refer to 3.5) that describes the handshaking process on the control channel and the flow of messages within different unicast, multicast and broadcast scenarios.

\subsubsection{Proposed MAC Radio Architecture}

Vehicles are equipped with two transceivers working simultaneously. The first transceiver is connected to an omnidirectional antenna configured on channel 178 and serves as a dedicated control channel. Vehicles in the same range listen and transmit control data on this channel and any change in the vehicle network topology is directly sent to inform neighboring vehicles to update their CNST. Vehicle's contention for this channel is based on CSMA/CA as defined by IEEE802.11p standard. However unlike the IEEE standard, only control frames are sent on this channel ensuring short messaging (50B) and short medium reservation. Beacons (Vehicle ID, Speed, and Position) and control frames for frequency selection (Adjusted RTS and CTS) are only sent on the dedicated control channel.

The second transceiver is a cognitive radio capable of hopping and adjusting between different frequencies ranging from the ISM and UNII-3 band up to DSRC band (5.725 $\mathrm{GHz}$ - 5. $925 \mathrm{GHz}$ ). The cognitive radio is solely used for transmitting and receiving data. Moreover when neither transmitting nor receiving data, the cognitive radio senses the ISM and UNII-3 band for noise and interference levels on each particular channel 
and selects the best channel to transmit on. There isn't any medium contention on this channel (contention-free) as the other transceiver (control channel) would reserve a particular service channel and announce it to all neighboring vehicles. By updating the vehicle's CNST, collisions between vehicles are avoided and no two vehicles are transmitting and receiving at the same time on same channel within the same range. In Brief, the intelligence and medium contention lies on the control channel with short messages preserving the channel from contention while the actual data sending (large frames) is done in frame bursts on the service channels based on the QoS Access Category.

\subsubsection{Supported Frames and Tables in the Proposed Protocol}

MQOG requires the use of special control frames to help in the negotiation process between vehicles and to ensure the collision -free transfer of information. Those are the only frames sent on the dedicated control channel and characterized by being short messages (50B). They are responsible for establishing the communication link between the transmitter and receiver for the actual data transmission on the service channel.

\begin{tabular}{|c|l|l|}
\hline $\begin{array}{c}\text { Vehicle } \\
\text { ID }\end{array}$ & $\begin{array}{l}\text { Vehicle } \\
\text { Velocity }\end{array}$ & $\begin{array}{l}\text { Vehicle } \\
\text { Position }\end{array}$ \\
\hline
\end{tabular}

(a)

\begin{tabular}{|l|l|l|}
\hline $\begin{array}{l}\text { Selected } \\
\text { Channel }\end{array}$ & $\begin{array}{l}\text { Access } \\
\text { Category }\end{array}$ & Transmitter \\
Physical Address \\
\hline
\end{tabular}

(c)

\begin{tabular}{|l|l|l|}
\hline $\begin{array}{l}\text { Handoff to } \\
\text { UNII-3 or } \\
\text { ISM Band }\end{array}$ & $\begin{array}{l}\text { Access } \\
\text { Category }\end{array}$ & Receiver \\
Physical Address \\
\hline
\end{tabular}

(e)

\begin{tabular}{|l|l|l|}
\hline $\begin{array}{l}\text { Channel } \\
\text { Selections }\end{array}$ & $\begin{array}{l}\text { Access } \\
\text { Category }\end{array}$ & $\begin{array}{l}\text { Receiver } \\
\text { Physical Address }\end{array}$ \\
\hline
\end{tabular}

(b)

\begin{tabular}{|l|l|l|}
\hline $\begin{array}{l}\text { Selected } \\
\text { Channel } \\
\text { for Handoff }\end{array}$ & $\begin{array}{l}\text { Access } \\
\text { Category }\end{array}$ & $\begin{array}{l}\text { Receiver } \\
\text { Physical Address }\end{array}$ \\
\hline
\end{tabular}

(d)

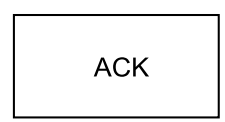

(f)

Figure 3.2: Control Frames Used on Dedicated Control Channel

- Beacon Management: (Fig.3.2a) Periodically sent by each vehicle to the neigh- 
boring vehicles within the range and it conveys the Vehicle ID, Velocity and Position.

- Adjusted-RTS : (Fig.3.2b) It is sent at the beginning when a vehicle requests to send any new data (safety or nonsafety). This frame replaces the known RTS frame by adding channel selection parameters which shows the assessed channels for transmission. Moreover, it has a unique QoS Access Category Level to prioritize data.

- Adjusted-CTS: (Fig.3.2c) It is the reply to the transmitter's request to send. This is analogous to the known CTS packet with the differences of adding a selected channel which would be the agreed channel between transmitter and receiver for best channel conditions for transmission and the QoS Access Category Level.

- Request-to-Handoff : (Fig.3.2d) It is a new Control Frame. This frame is triggered once a frame with high priority is required to be sent (example safety message) while all other channels are occupied by lower priority transmissions. The request to handoff selects the channel occupied with lowest priority and sends a request to the transmitting vehicle to handoff to another ISM or UNII-3 channel since this channel would be reserved for the incoming more prior transmission.

- Handoff-to-ISM or UNII-3: (Fig.3.2e) It is also a new Control Frame. This frame is sent from the transmitter in an ongoing communication to the receiver on the dedicated control channel. This informs the receiver to switch to the following ISM or UNII-3 band as the channel being used would be granted to a more prior transmission.

- ACK: (Fig.3.2f) Sent in response to the Request-to-Handoff frame to inform the vehicle that the request was received and the other vehicle is initiating with the handoff process. 
Channel Neighbor State Table(CNST):As shown in Fig. 3.3, CNST stores the ongoing neighboring communications between neighboring vehicles in every vehicle in its database. Neighboring vehicles continuously update their channel transmission by sending control frames on the dedicated control channel. CNST stores the following information for every ongoing transmission:

\begin{tabular}{|l|l|l|l|}
\hline $\begin{array}{l}\text { Frequency } \\
\text { Channel }\end{array}$ & $\begin{array}{l}\text { Access Category } \\
\text { Priority Level }\end{array}$ & $\begin{array}{l}\text { Countdown Duration } \\
\text { of Transmission }\end{array}$ & $\begin{array}{l}\text { Reservation } \\
\text { Field }\end{array}$ \\
\hline
\end{tabular}

Figure 3.3: Channel Neighbor State Table (CNST)

- Frequency Channel: The frequency channel being used by the corresponding communication link.

- AC Priority Level: The Access Category of the data being sent on that channel highlighting the QoS and the nature of transmission as unicast or multicast/broadcast.

- Duration Value: The duration of the ongoing transmission and it is a countdown timer.

- Reserved: This field shows the Vehicle ID reserving the channel either for handoff or for an incoming transmission. For handoff purposes, this is triggered when the Request-to-Handoff control frame is sent reserving the channel for a more prior communication ensuring a First Come First Serve (FCFS) basis.

Quality of Service:In our proposed protocol we define 6 access categories rising from the different Vehicular QoS needs. The Access Categories are shown in Table 3.1 ranging from lowest to highest priority and are divided into 3 main categories: safety, 
nonsafety realtime, and nonsafety non realtime. Examples are also presented to show the different applications involved in a vehicular scenario.

Table 3.1: QoS Different Category Levels

\begin{tabular}{lcl}
\hline Access Categories & Priority & Examples \\
\hline NonSafety Non Real Time (Unicast) & 1 & Web Surfing/Sending Emails \\
NonSafety Non Real Time (Broadcast/Multicast) & 2 & Downloading EMaps from RSU \\
NonSafety Real Time (Unicast) & 3 & Voice Conversation \\
NonSafety Real Time (Broadcast/Multicast) & 4 & Video/Voice Conference \\
Safety (Unicast) & 5 & Lane Change or Wrong Way Warning \\
Safety(Broadcast/Multicast) & 6 & Warning Messages (Accident, Icy \\
& & Road, Oil Stain, Sudden Breaking...) \\
\hline
\end{tabular}

\subsubsection{Overall System Operation}

As shown in Fig.3.4, the vehicle can operate in three different modes. In the idle mode, the control channel exchanges control info and updates the CNST Table while the cognitive radio senses unlicensed and licensed bands for channels for reliable transmission. By updating the vehicle's CNST, collisions between vehicles are avoided and no two vehicles are transmitting and receiving at the same time on same channel within the same range. In the pre-transmission mode, the channel negogiation occurs on the control channel while the dynamic channel allocation algorithm occurs on the cognitive radio. Finally, during the transmission mode, the cognitive radio sends the data on the selected channel while the control channel keeps on exchanging control frames and updating the CNST Table. 


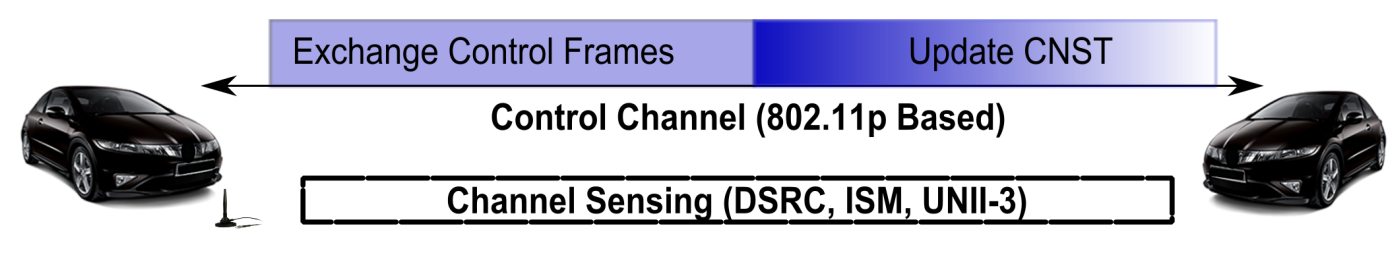

Cognitive Radio

(a) Idle Mode (Nothing to Send)

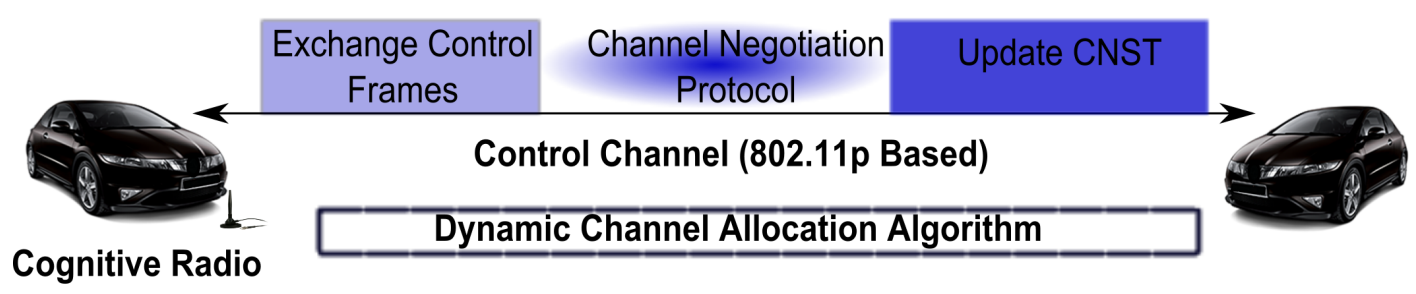

(b) Initiating Transmission Mode (Setting up the Connection)

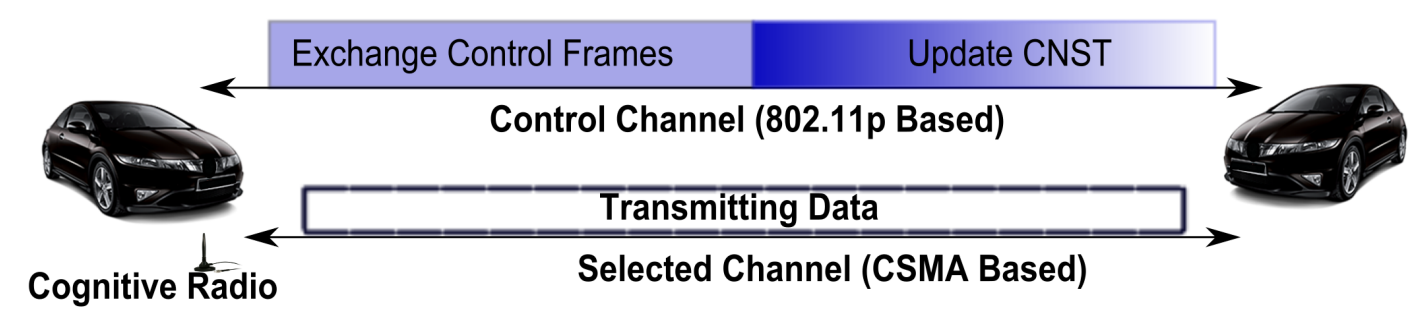

(c) Transmission Mode (Transmitting Data)

Figure 3.4: Proposed Protocol's Modes of Operation

\subsection{Dynamic Channel Allocation Algorithm}

The Dynamic Channel Allocation Algorithm selects the best available channel with low interference and noise levels. The flowchart is shown in Fig.3.5 and it works as follows:

If the Cognitive Radio Assessment reveals an available DSRC channel with optimum conditions then it is directly selected for the incoming transmission. However, if all available DSRC channels are being used by neighboring vehicles then an assessment for the level of noise and interference on each channel is made. If at least one channel is above the threshold then this channel is selected and checked by comparing it with the CNST. This channel is then reserved by the vehicle for the upcoming transmission.In our 


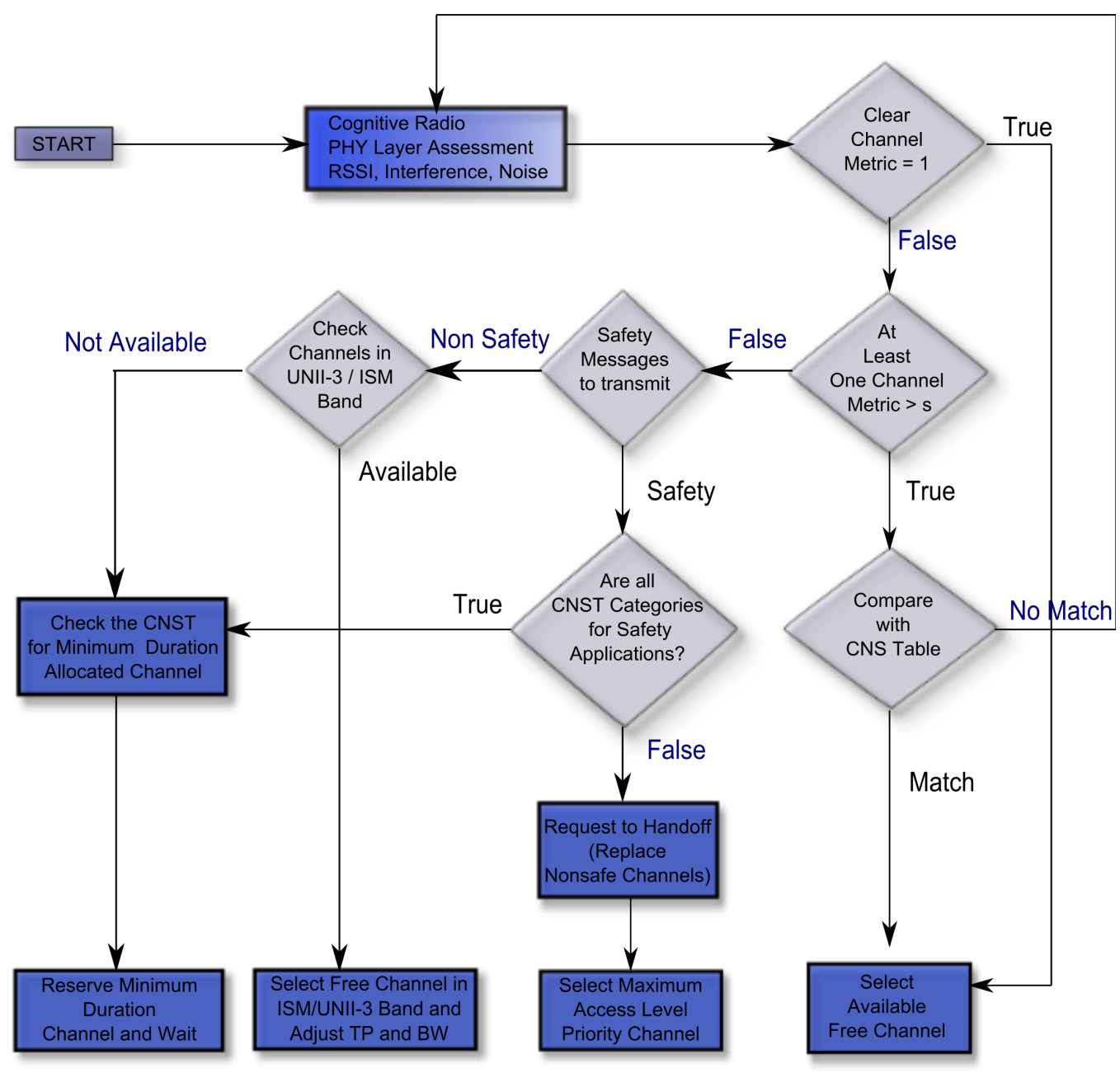

Figure 3.5: Dynamic Channel Allocation Protocol Flowchart

simulations, we set the threshold s to 0.5 considering 1 as clear channel assessment. This threshold is the RSSI (Received Signal Strength Indicator) which is proven to be suitable for wireless networks as it has also been used in Air Magnet Site Surveying tool[40]. As shown in Table 3.2, the RSSI of a selected channel should be greater or equal than 15 . This number indicates that the channel has a Signal to Noise Ratio (SNR) above 33 dB and a strong signal strength.

If all channels suffer from high interference and noise levels then the QoS of the 
message to be sent is investigated:

If the message to be sent is safety then CNST is checked to assess if all occupied channels belong to safety transmissions. If this is the case, then the vehicle has to wait for the end of the first transmission. If neighboring vehicles are transmitting non safe data then the vehicle sends a request to handoff and safety transmission would replace nonsafe transmission on the DSRC channel while nonsafe transmission are handed off to an ISM or UNII-3 Channel.

If the frames to be sent are non safe messages then transmission can occur on the ISM or UNII-3 since it is not a critical message. Unlicensed Channels are checked for the interference and noise levels and once a channel with good conditions is assessed, it is then directly selected for transmission. Before initiating transmission, adjusting Transmit Power (TP) is necessary to ensure the abidance to RF regulations of the local regulatory entity. If all ISM/UNII-3 bands were suffering from high interference levels then after checking CNST the vehicle reserves the channel the first to be vacant.

Table 3.2: Channel Assessment Properties [8]

\begin{tabular}{lcccc} 
RSSI & Rx Sensitivity Threshold & Signal Strength & SNR & Signal Quality \\
\hline 30 & $-30 \mathrm{dBm}$ & $100 \%$ & $70 \mathrm{~dB}$ & $100 \%$ \\
25 & $-41 \mathrm{dBm}$ & $90 \%$ & $60 \mathrm{~dB}$ & $100 \%$ \\
20 & $-52 \mathrm{dBm}$ & $80 \%$ & $43 \mathrm{~dB}$ & $90 \%$ \\
21 & $-52 \mathrm{dBm}$ & $80 \%$ & $40 \mathrm{~dB}$ & $80 \%$ \\
15 & $-63 \mathrm{dBm}$ & $60 \%$ & $33 \mathrm{~dB}$ & $50 \%$ \\
10 & $-75 \mathrm{dBm}$ & $40 \%$ & $25 \mathrm{~dB}$ & $35 \%$ \\
5 & $-89 \mathrm{dBm}$ & $10 \%$ & $10 \mathrm{~dB}$ & $5 \%$ \\
0 & $-110 \mathrm{dBm}$ & $0 \%$ & $0 \mathrm{~dB}$ & $0 \%$
\end{tabular}




\subsection{Channel Negotiation Algorithm}

In this section, we discuss the channel negotiation protocol within different communication scenarios (unicast and multicast/broadcast) to ensure the correct transmission sequence of control frames to establish the communication link between the transmitter and receiver(s). Considering a unicast transmission, a vehicle after running the dynamic channel algorithm protocol is left with two cases either an available DSRC Channel or High level of Interference on DSRC so switching to an ISM or UNII-3 channel. For the former, the negotiating protocol on the dedicated control channel is done by the following steps:

1. Transmitter sends an Adjusted-RTS with the selected list of frequencies to the receiver

2. Receiver replies with an Adjusted-CTS with an agreed upon channel.

3. Transmitter sends again the same CTS as a CTS-to-Self Frame for all neighboring vehicles to solve the hidden node problem.

4. Neighboring vehicles update their CNST and record the transmission on the allocated channel with the countdown duration defined.

5. Transmission of data occurs on the second transceiver after both transceivers tune to same frequency.

Regarding the case of switching to an ISM or UNII-3 band, the negotiating protocol on the dedicated control channel is done by the following steps:

1. Transmitter checks CNST for all transmissions on all channels and selects the lowest priority channel (NonSafety Only). 
2. Transmitter sends a Request-to-Handoff for the transmitting vehicle on the channel to replace it with its incoming transmission as it is more prior.

3. All neighboring vehicles first update their CNST by filling the Request-to-HandoffField to reserve the channel for this particular transmission.

4. Receiver replies with Handoff-to-ISM frame to switch its communication link to the ISM or UNII-3 band then sends another ACK to the transmitter.

5. Transmitter now precedes as unicast with available DSRC channel (same as first case)

The second case to be considered is sending multicast/broadcast frames. The negotiating protocol on the dedicated control channel is done by the following steps:

1. Transmitter sends an Adjusted-RTS with the selected list of frequencies to many receivers.

2. All receivers check their CNST for that particular channel frequency with the corresponding access category. If a vehicle had the channel occupied with a certain transmission then it checks the QoS AC (Access Category) of this particular transmission.

- If $\mathrm{AC}$ is less prior then it will defer the old transmission and reserve it for the incoming transmission.

- If AC was more prior then it won't defer but wait for the completion and buffer the new transmission.

3. Transmitter sends data on the corresponding service channel. 


\subsection{Conclusion}

In this work, I proposed a new Multichannel QoS Cognitive MAC (MQOG) tailored for vehicular communications. Channel sensing is done prior to transmission and messages are always sent on the best available channel to mitigate high interference and multipath problems. Moreover, this protocol ensures QoS by granting safety messages higher priority of accessing the medium over data messages by making use of the ISM and UNII bands which precede the DSRC Band. Those features make the proposed protocol MQOG more suitable for VANET environments. 


\section{Chapter 4}

\section{Enhanced Multichannel QoS Cognitive MAC for VANets (EMQOG)}

In this chapter I propose a MAC enhancement scheme for our already proposed MQOG protocol. The new enhancement scheme is MoByToP (Mobility-Based Dynamic Transmit Opportunity) added to the medium reservation phase. I first start by outlining the motivation behind our work then I explain in details MoByToP operation with its different parts.

\subsection{Motivation}

MQOG mainly addresses the issue of granting safety messages a reliable delivery in congested environments with high interference by making use of the free ISM and UNII-3 bands already available. Moreover its architecture with one channel dedicated for control using a carrier sense contention and the other for data transmission showed improvements 
in throughput compared to other already existing MAC protocols (refer to chapter 5). However MQOG doesn't address the issue of providing fair access between vehicles for medium contention and link reservation. Vehicles in a vehicular network cannot be treated equally under all circumstances. In this work, we investigate three conditions where vehicles' medium contention should be looked at more closely.

First, cars moving with high speeds cannot be looked upon the same way as stationary cars or cars moving with low speed since high speed cars have a link expiration time set by their transmission range. As shown in Fig.4.1(a), Car A is communicating with Cars B and C. However, since Car B is moving with high speed compared to Car A, it is getting out of range of Car A. In this case, we have to send as many frames between Car A and B before the link breaks. Car C can meanwhile hold its communication with A for few seconds since the communication link between Cars B and A is critical and more prior at that time.

Secondly, VANet imposes life-critical safety frames which should be transmitted and given the highest priority unlike regular networks where we only have realtime and nonrealtime applications. Cars sending safety frames must be able to access the medium and transmit a burst of frames whereas cars sending data should reserve the medium for less time. As shown in Fig.4.1(b), Car B is sending safety frames whereas Car C is sending non realtime frames. In this case, those two communications cannot be treated equally for medium contention and link reservation. The safety application should be able to contend faster for the medium and preserve it for a longer time since its frames are of life-critical, time-sensitive, and delay-nontolerant nature.

Thirdly, as VANet is considered a high multipath environment, some vehicles may have weak signal reception due to non line of sight communication. So those vehicles would have a very low download data rate. In this case, the transmitting vehicles should reserve the medium longer than cars transmitting at a faster data rate. As shown in 
Fig.4.1(c), Car B has a low signal strength reception and thus its download data rate is $512 \mathrm{Kbps}$ whereas Car $\mathrm{C}$ has a better signal strength reception and its transmit data rate is $2 \mathrm{Mbps}$. In this case, if we treated equally both cars than Car A would be sending more traffic to Car C than Car B. To alleviate this problem, we allocate a longer link reservation time for Car B so that Car B is receiving frames with the same data rate but within a longer interval than Car C.

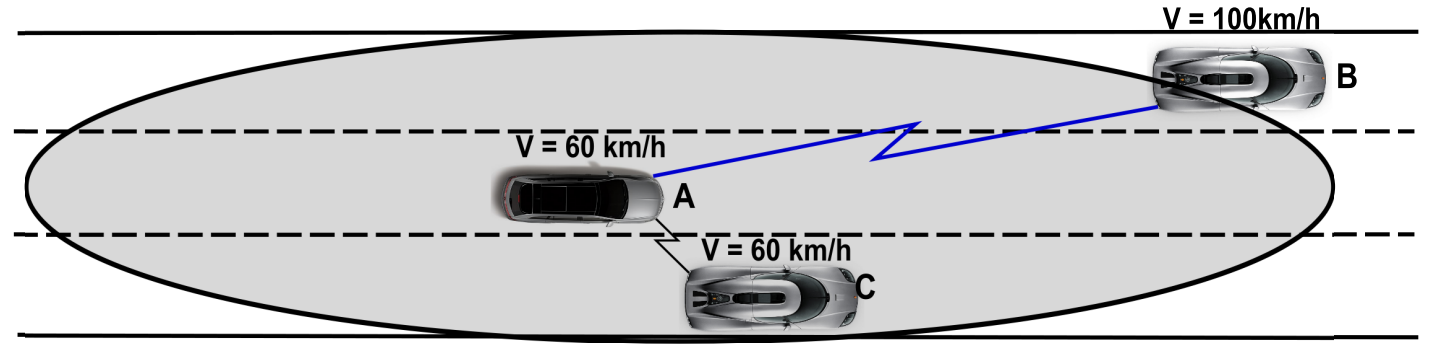

(a) Out of Communication Range

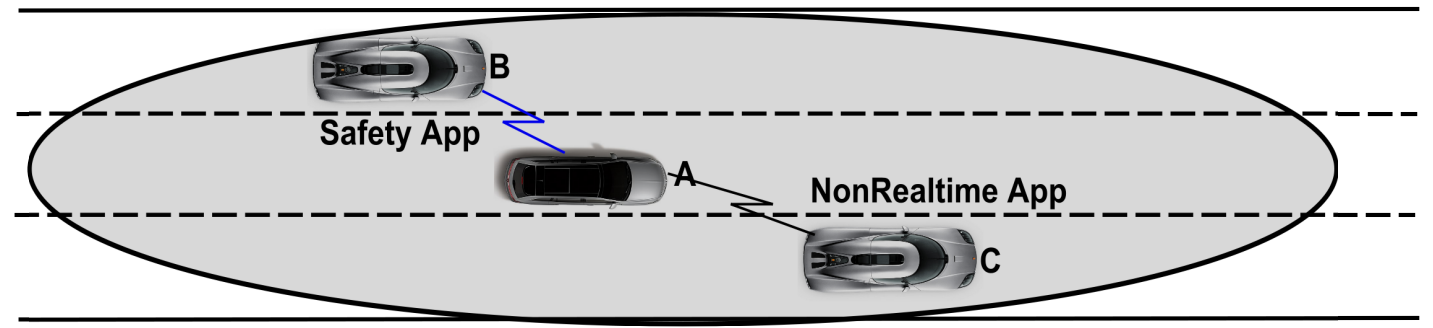

(b) Different QoS Categories

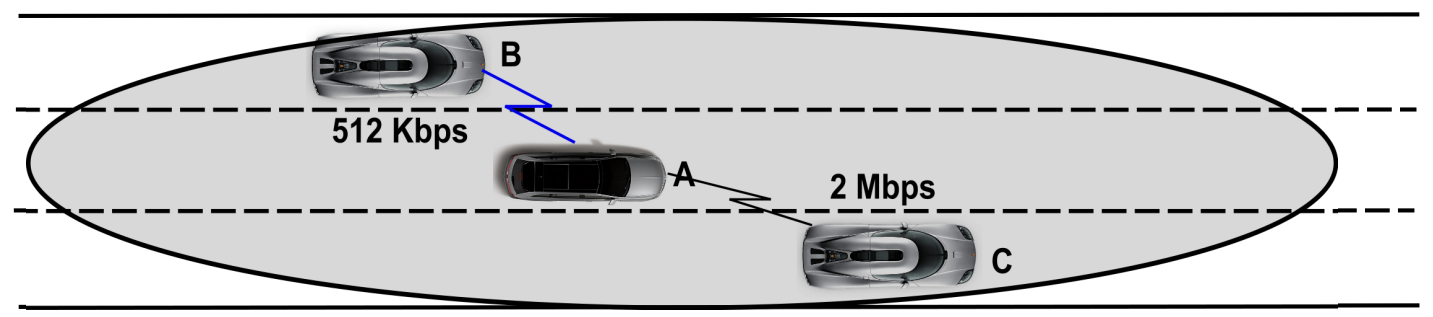

(c) Fluctuating RSS leading to Different Transmission Data Rates

Figure 4.1: MAC Issues in VANets

Those cases urged us to reconsider designing the MAC for VANets taking into account those three aspects. MoByToP mechanism proposed particularly addresses those issues. 


\subsection{Transmit Opportunity (TXOP) and Related Work}

\subsubsection{TXOP Defined}

The IEEE 802.11e amendment already incorporated by IEEE802.11p WAVE standard defined the MAC layer methods needed to meet the QoS requirements for time-sensitive applications and added a new coordination function known as HCF (Hybrid Coordination Function)[31]. DCF (Distributed Coordination Function) which was used in 802.11 allow for a radio to transmit a single frame (refer to 2.1.2). After transmitting the frame, the 802.11 station must contend for the medium again before transmitting another frame. HCF defines the ability for a radio to send multiple frames. When a HCF-compliant radio contends for the medium, it receives an allotted amount of time to send frames. This period of time is called Transmit Opportunity (TXOP). During this TXOP, an 802.11 radio may send multiple frames in what is called frame burst. During the frame burst, a short interframe space (SIFS) is used between each frame to ensure that no other radios transmit during the frame burst. A comparison between original 802.11 and 802.11e modes of operation is shown in Figure 4.2.

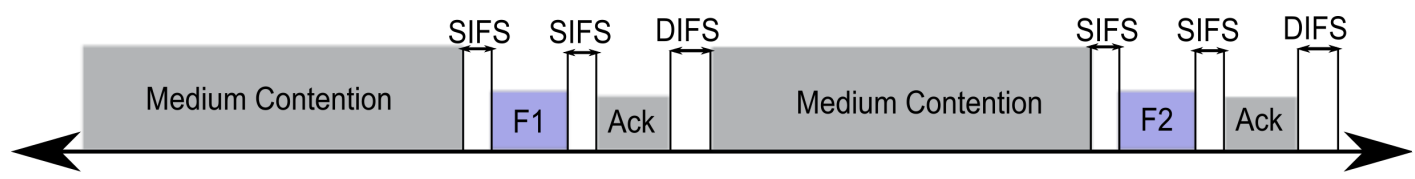

(a) 802.11 Packet Transmission Operation

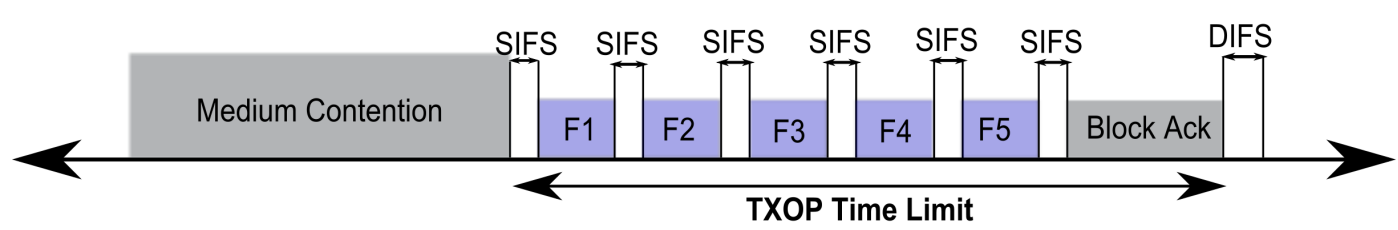

(b) 802.11e EDCA Packet Transmission Operation

Figure 4.2: 802.11 Different Transmit Operation Mechanisms

Moreover, HCF defines four Access Categories based on the different QoS Categories. 
For each access category, there exists a unique CW (Contention Window), AIFSN (Arbitraty Interframe Space Number) and TXOP value. Table 4.1 highlights the different access categories and corresponding TXOP values. Frames with highest-priority access category have the lowest backoff-values and therefore are more likely to get a longer TXOP. However what makes 802.11e unsuitable for VANets is that the TXOP allocated doesn't consider mobility nor safety QoS. The four QoS categories don't include a safety category which has to be incorporated and granted the highest priority considering the life-critical nature of the corresponding VANet applications.

\begin{tabular}{lll} 
Table 4.1: 802.11e EDCA & Parameter Sett \\
\cline { 2 - 3 } Traffic Type & AC & TXOP limit \\
\hline VOIP & 0 & $3,264 \mathrm{~ms}$ \\
Video & 1 & $6,016 \mathrm{~ms}$ \\
Best Effort & 2 & 0 \\
Background & 3 & 0 \\
\hline
\end{tabular}

\subsubsection{Related Work Using TXOP}

In general, MAC layer enhancements can be tackled in two different approaches: The first approach which is more commonly used is to improve the efficiency of the contention phase by enhancing the contention window and slot time[41-45]. Whereas the second approach improves the efficiency of the medium reservation phase after gaining access to the medium by transmitting a frame burst (more than one frame).

The first approach which has been extensively used in different Ad Hoc and wireless networks has been carefully studied in [41-45]. Lots of work have been done to optimize the contention window by using mathematical models [41] or tuning the backoff algo- 
rithm with differentiated channel access [42-43]. For vehicular networks, the authors in [44-45] proposed medium contention schemes to address mobility in V2I [44] and V2V [45]. In [45], Alasmary and Zhuang tackle the unfairness problem in $802.11 \mathrm{p}$ by proposing two dynamic contention window schemes. The authors address the mobility impact on the MAC Layer by adjusting the medium contention phase and adapting it to vehicles' velocities or number of neighboring nodes. The first scheme provides dynamic level of service priority via adaptation to the number of neighboring nodes, while the second based on node relative speed. It has been shown through simulations that those schemes alleviate network performance degradation due to high mobility. The downside of those schemes is that they only consider mobility while overlooking other factors such as QoS of the transmitted frames and the downloaded data rate. On the other hand, in my thesis, I solve the contention issue by integrating mobility and QoS to the medium reservation phase instead of the contention phase.

Regarding the second approach, we look into the MAC layer from the medium reservation perspective i.e. after a node gains access to the medium. Most of the work already done [46-48] had been to enhance TXOP for non-vehicular scenarios targeting enhancements in the MAC layer for the IEEE 802.11e standard. In [46-48] work has been done to enhance the TXOP mechanism of the IEEE 802.11e standard by introducing a dynamic TXOP mechanism. In [47], Guo, Chen and Pei target the fairness problem by adjusting the transmit data rate of different nodes with the TXOP interval. Moreover, they propose a scheme that utilizes the collision times to estimate contention degree before transmission and then adjust TXOP accordingly. In [48], Majkowski and Palacio propose another dynamic TXOP scheme that aims to define the dynamic rules for TXOP duration considering the corresponding data rate, MSDU (MAC Service Data Unit) size and buffer size. Although those schemes improve the performance of the 802.11e MAC layer, however they are not suitable for vehicular networks as they don't consider the 
cars mobility and the frequent network topology changes with many connections and disconnections.

In this chapter, I emphasis the importance of medium reservation in vehicular networks and thus I introduce MoByToP. MoByToP considers the QoS of the corresponding data, the mobility of the vehicles and the transmit data rate hence enhancing the MAC in VANets. To best of our knowledge, MoByToP is the first dynamic TXOP mechanism for vehicular communications.

\subsection{A Proposed Dynamic TXOP (MoByToP): De- sign}

Unlike multimedia networks where prioritizing traffic only depends on QoS (audio, video and voice), MobyToP mechanism enhances the medium reservation phase by prioritizing traffic according to vehicular speed, download data rate, and QoS. MoByToP first calculates a ceiling for the maximum TXOP attainable by considering the available buffer size, maximum data rate and length of the MSDU frame. Hence this duration is the maximum time allowed for a single burst of frame transmissions between two vehicles considering technological limitations. Secondly, MoByToP assesses the QoS of the traffic to be submitted as it can belong to safety, realtime or non-realtime applications. Safety applications are given the highest priority followed by realtime then nonrealtime. To account for fairness in link reservation, MoByToP then adjusts the TXOP duration based on the average transmit data rate as vehicles with low RSS (Received Signal Strength) levels receives data slower than others. Hence, MoByToP is taking account the high interference and multipath in vehicular environments. Finally to incorporate mobility in an integrated manner, MoByToP calculates the time needed for two vehicles to get out of communication range given their positions and speeds and then assess if this time is 
less than the ceiling TXOP. If this is the case, it will be assigned the new TXOP. Hence two vehicles going out of communication range are prioritized over others as they exchange a longer frame burst and hold the medium for a longer duration. In the following subsections, we explain MoByToP mechanism in details.

Maximum Duration of a Frame Burst: We define CeilToP as the maximum duration for a frame burst. CeilToP has a physical limitation depending on the buffer, MSDU size and transmit data rate. In [13] authors define the maximum TXOP attainable by the following formula:

$$
\text { CeilToP }(s)=\frac{L(\text { bits }) \times N}{R(\text { bits } / s)}
$$

Where $\mathrm{L}$ is the length of the MSDU frame, $\mathrm{N}$ is the maximum number of packets in the buffer and $\mathrm{R}$ is the transmit data rate (bps). The formula shows that maximum TXOP is directly proportional to the number of packets transmitted and the buffer size and indirectly proportional to the transmit data rate. So to achieve same TXOP duration with small $\mathrm{R}$ (data rate) values, we need to increase $\mathrm{N}$ hence transmitting more MSDU frames. CeilToP may have different values depending on the average data rate and the technology being used. In our simulations, we set CeilToP to 6,032 ms (same maximum value as the IEEE 802.11e). This number was shown to be suitable for the VANet scenario after performing extensive simulations and testing with other values.

Accounting for Different Quality of Service Categories:In vehicular networks, safety application traffic should be prioritized before voice, audio and video due to its life critical nature. MoByToP incorporates QoS by granting frames with higher priority a longer frame burst thus leading to a higher TXOP interval. This can be achieved after applying the following equation: 


$$
Q_{-} \operatorname{TxoP}(s)=\frac{n}{6} \times \operatorname{CeilToP}(s)
$$

Where $\mathrm{n}$ is the priority number of the corresponding QoS Access Category and CeilToP is the maximum TXOP achieved.

Download Data Rate: Vehicular networks are considered high multipath environments with dynamic delay spreads due to multiple reflections, scattering, diffraction and refraction. This type of environment causes fluctuating RSS levels. Moreover, communication links might be Non-LOS (line of sight) due to the presence of many obstacles between the two communicating vehicles. This would weaken the signal propagation and reception. Since the signal strength is directly proportional to the data rate received[49], some vehicles might be receiving data with very slow data rates while others might be much faster. Therefore, the unfairness in terms of throughput is unavoidable even if stations have the same QoS level (AC). To alleviate the fairness problem, MoByToP manages to assess the download data rate before assigning the corresponding TXOP. Thus, it aims to give a longer duration (longer frame burst) for vehicles with low download data rates while a shorter duration for vehicles with faster data rates. In [47], the authors propose a mechanism to consider the transmit data rate and TXOP adjustment. This mechanism is applied to a wireless network (not vehicular) with heavy load conditions. MoByToP uses part of that mechanism while integrating it with mobility and QoS enhancements. To mathematically model the problem, authors in [47] assign a coefficient Data Rate Ratio (DRR) to adjust the TXOP of different stations. The coefficient is defined as:

$$
D D R=\frac{\text { DataRate }(\text { bits } / s)}{\text { Average_DataRate }(\text { bits } / \text { s })}
$$

Where DataRate is the current download data rate and Average_DataRate is a predefined value for the average data rate in a Vehicular network set to be $12 \mathrm{Mbps}[50]$. 
Using DRR to calculate the new TXOP, we set:

$$
R Q_{-} T x o P(s)=\frac{Q_{-} T x o P(s)}{D D R}
$$

where Q_TXOP is the adjusted QoS Data Rate (defined in previous subsection)

MoByToP uses this equation as part of its mechanism and thus the TXOP is adjusted based on the average transmit data rate granting slow transmissions a longer frame burst and fast transmissions a slower frame burst. This would provide fairness to the vehicular environment.

LOP (Link Out Of Range Prediction): As shown in Figure 3, Vehicle A is communicating with both vehicles $\mathrm{B}$ and $\mathrm{C}$. Vehicle $\mathrm{C}$ is driving in the same direction as vehicle $\mathrm{A}$ however vehicle $\mathrm{B}$ is driving out of range of $\mathrm{A}$. As the medium is alternating between $\mathrm{A}-\mathrm{B}$ and $\mathrm{A}-\mathrm{C}$, it is better at this point to transmit longer frame bursts between A-C before the link breaks thus allocating for this communication link a longer TXOP. Although this would create delay in the communication between A-B but as long as the two cars are driving in tandem, they can always compensate for that delay in the coming seconds.

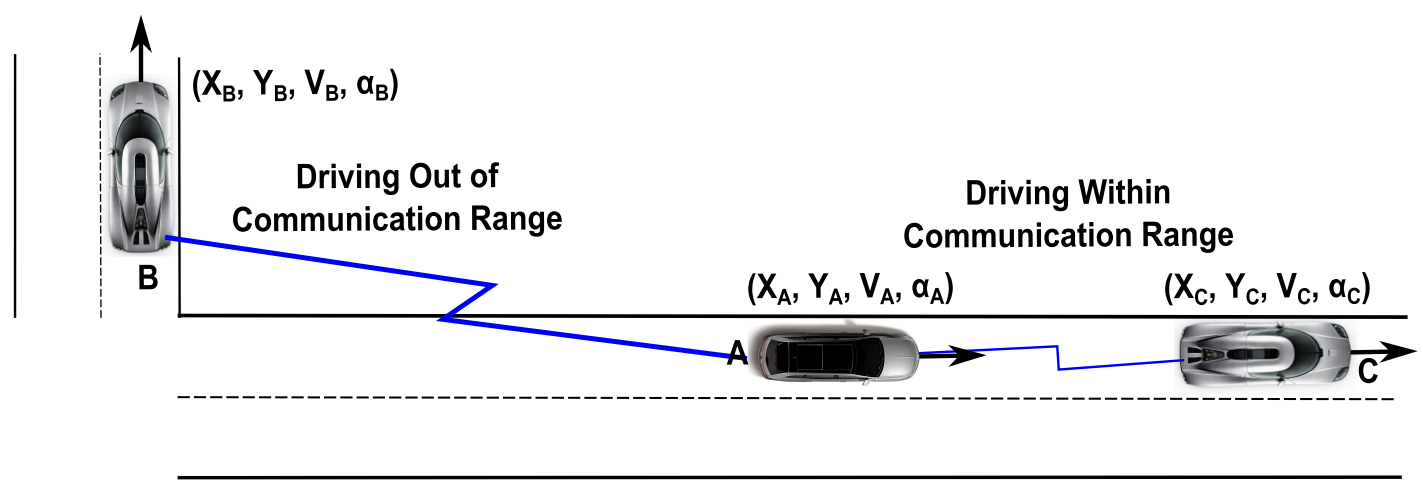

Figure 4.3: Comparison between driving within and out of communication range 
The following assumptions are important for the calculation of LOP and then integrating it in the TXOP operation.

1. Each vehicle has a bidirectional communication with any neighbor vehicle. The theoretical communication Range set by IEEE 802.11p is 1000m [50]. In[51], authors evaluated the performance of IEEE 802.11p PHY layer and the actual range measured was $700 \mathrm{~m}$ in a real life scenario. So vehicles within $700 \mathrm{~m}$ are capable to initiate and maintain a communication link and thus called adjacent vehicles.

2. Each vehicle is set to transmit a periodic beacon frame. The beacon frame contains the vehicle ID, position, speed and direction angle.

3. The speed, position and direction angle are assumed to be constant for the time of link calculation which is not supposed to take more than 1 second. In case of any change, this would take account in the next calculation as the mechanism is highly dynamic and implemented before every frame transmission.

4. Each vehicle is capable of calculating its direction angle from its previous position. The process of calculating the direction angle is depicted in Figure 4.4. As car A is moving from position 1 to position 2, the direction angle is calculated as the inverse of the tangent of $\delta y\left(\mathrm{y}_{2}-\mathrm{y}_{1}\right)$ over $\delta x\left(\mathrm{x}_{2}-\mathrm{x}_{1}\right)$.

5. LOP is calculated for a one hop communication link between two cars.

To calculate LOP, we set 2 adjacent vehicles $\mathrm{A}$ and $\mathrm{B}$ moving with velocities $\vec{V}_{A}$ and $\overrightarrow{V_{B}}$ respectively along a stationary Cartesian Coordinate System with orthogonal unit vectors $\hat{x}$ and $\hat{y}$ along the $\mathrm{X}$ and $\mathrm{Y}$ axes respectively. As $V x=V \cos \alpha$ and $V y=V \sin \alpha$ then 


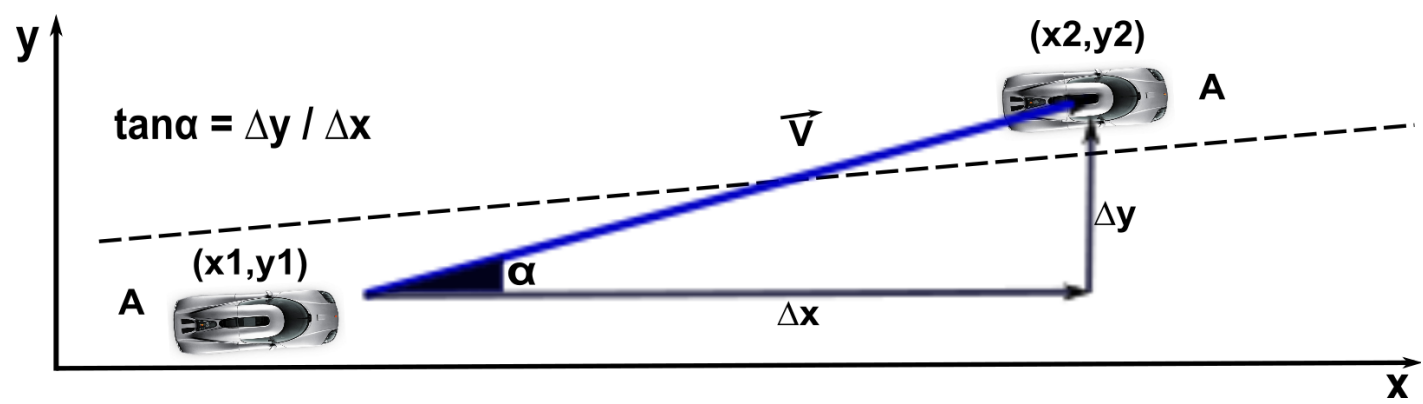

Figure 4.4: Calculating the Direction Angle

$$
\begin{aligned}
& \overrightarrow{V_{A}}=\left(V x_{A}, V y_{A}\right) \\
& \overrightarrow{V_{B}}=\left(V x_{B}, V y_{B}\right)
\end{aligned}
$$

The parametric equations of motion for both Cars A and B are:

$$
\begin{aligned}
& \overrightarrow{O A}=\left(X_{A}+V x_{A} t, Y_{A}+V y_{A} t\right) \\
& \overrightarrow{O B}=\left(X_{B}+V x_{B} t, Y_{B}+V y_{B} t\right)
\end{aligned}
$$

Let:

$$
\begin{aligned}
& V x_{B}-V x_{A}=\triangle V x \quad X_{B}-X_{A}=\triangle X \\
& V y_{B}-V y_{A}=\triangle V y \quad Y_{B}-Y_{A}=\triangle Y \\
& \overrightarrow{A B}= \begin{cases}\left(X_{B}+V x_{B} t\right)-\left(X_{A}+V x_{A} t\right) & =\Delta X+\Delta V x t \\
\left(Y_{B}+V y_{B} t\right)-\left(Y_{A}+V y_{A} t\right) & =\Delta Y+\Delta V y t\end{cases}
\end{aligned}
$$

LOP is achieved when the distance between A and B is greater than $700 \mathrm{~m}$. Setting $\|\overrightarrow{A B}\|$ as the distance between $\mathrm{A}$ and $\mathrm{B}$, and solving for the time where the distance is greater than $\mathrm{R}$ would result in LOP. 


$$
\begin{gathered}
\|\overrightarrow{A B}\|^{2} \geq R^{2} \\
(\triangle X+\triangle V x t)^{2}+(\triangle Y+\triangle V y t)^{2} \geq R^{2} \\
t^{2}\left(\triangle V x^{2}+\triangle V y^{2}\right)+2 t(\triangle X \triangle V x+ \\
\triangle Y \triangle V y)+\left(\triangle X^{2}+\triangle Y^{2}\right)-R^{2} \geq 0
\end{gathered}
$$

This is a second degree equation in $\mathrm{t}($ time). Solving this equation will result in 2 roots a positive and a negative one. Since time is always positive we are only concerned with the positive root. Solving for $t$ we get:

$$
t=\frac{(\triangle V x \triangle X+\triangle V y \triangle Y)+\sqrt{\left(\triangle V x^{2}+\triangle V y^{2}\right) R^{2}-(\triangle V x \triangle Y-\triangle X \triangle V y)^{2}}}{\triangle V x^{2}+\triangle V y^{2}}
$$

By calculating Link Out of Range Prediction we are able to predict after how many seconds the link would break. If the link is breaking then we set TXOP to its maximum CeilTop and then reserve the medium the longest possible. Otherwise if LOP is larger than maximum TXOP, TXOP will be adjusted based on QoS and download data rate. In the next subsection, we explain the overall operation of MoByToP.

Overall Mechanism: MoByToP mechanism is applied before a vehicle transmits any packet. Algorithm 1 describes the detailed process that MoByToP goes through and all the steps involved in calculating the most efficient TXOP for a VANet scenario. 


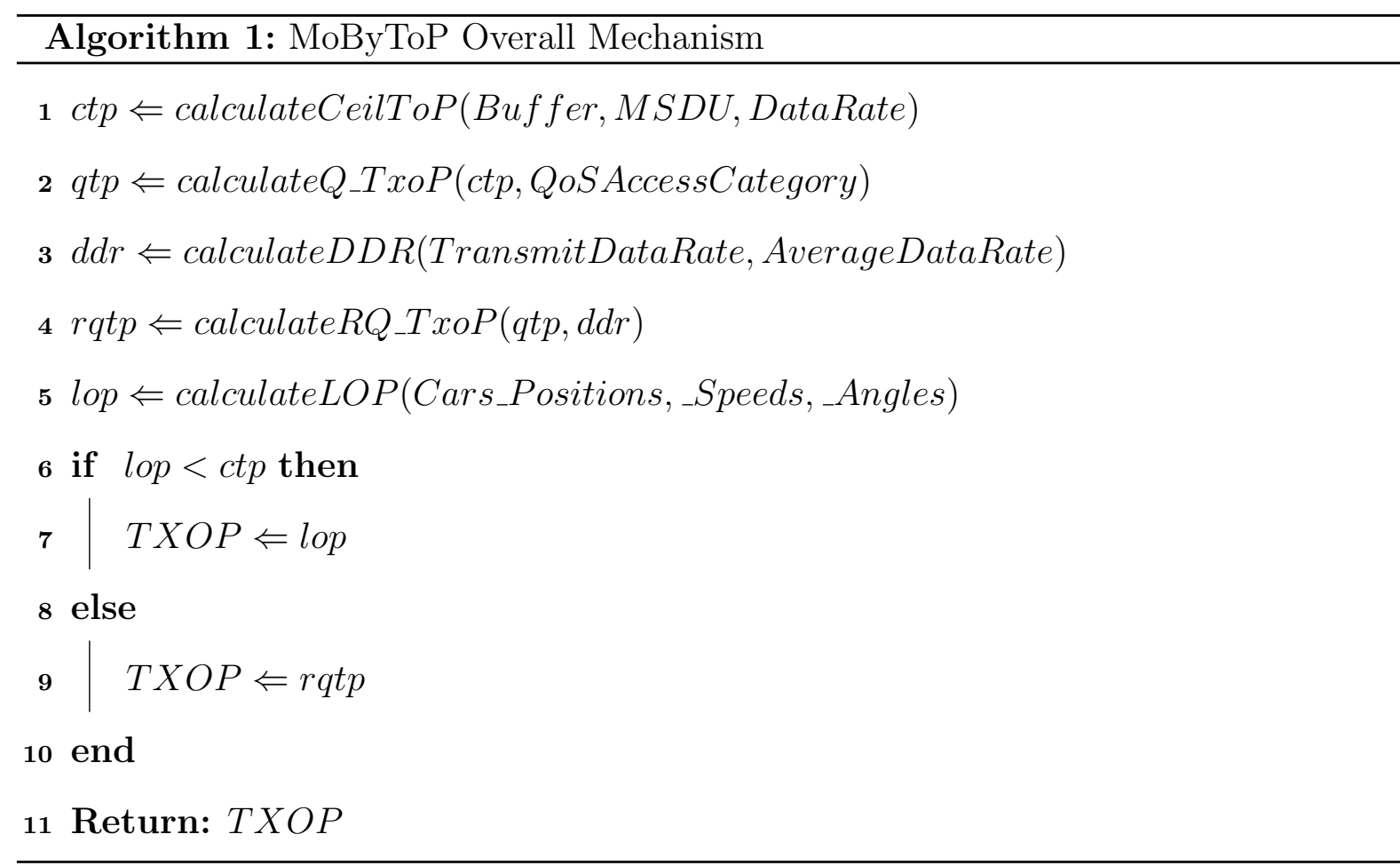

\subsection{Enhanced-MQOG: Integrating MoByToP Scheme with MQOG}

In this section, I am going to discuss the integration of MoByToP with MQOG (Chapter 3). Adding MoByToP scheme to MQOG is relatively straightforward and simple since both protocols are independent.

- MoByToP works on the medium reservation phase after a vehicle gains access to the medium whereas MQOG provides the architecture of MAC operation and the phase preceding gaining access to the medium.

- MQOG defines the modes while MoByToP defines the duration of transmission.

- MQOG provides fast reliable access to the medium, MoByToP provides fair access in holding the medium. 
Hence combining both MQOG and MoByToP result in EMQOG which is a fair and efficient MAC able to mitigate most MAC challenges in vehicular environments.

\subsection{Conclusion}

In this chapter, I proposed MoByToP, a MAC Layer mechanism, tailored for vehicular communications. MoByToP incorporates mobility, QoS and transmit data rate and integrates them into the MAC to mitigate VANet challenges. It uses beacon information to calculate LOP (Link Out of Range Prediction) and then uses it to circumvent link failure. Moreover, it ensures QoS by granting safety messages higher priority of reserving the medium over data messages. In addition, MoByToP adjusts the frame burst based on the RSS (Received Signal Strength) to create a fair effective sharing of resources and hence enhancing the system's overall throughput. Those features combined make MoByToP mechanism very suitable for VANets. 


\section{Chapter 5}

\section{Performance Evaluation of Our Proposed MAC Protocols}

In this chapter I evaluate both MQOG and EMQOG. Performance evaluation was carried out in OMNET++4.1 coupled with SUMO to generate real traffic mobility. Realistic maps were also selected from Ottawa by using the OpenStreetMap Project. Performance Evaluation metrics were captured and compared to 802.11p, 802.11e and DCA multichannel protocol(refer to 2.5.2).

\subsection{Simulation Environment Characteristics}

OMNET++: Discrete Event Network Simulator - To evaluate the proposed protocols, the implementation and simulation were done using OMNET ++ version 4.1 which is a discrete event network simulator composed of reusable intercommunicating models written in $\mathrm{C}++$. Making use of the software reuse, INETMANET framework is an already built in project that comprises many known networking layer protocols for MANets (Mobile Adhoc Network) and it was added to OMNET++ as part of the simulation[52]. 
Simlation of Urban MObility (SUMO): Regarding the traffic simulation and mobility, I used SUMO which is a microscopic traffic simulator[53]. SUMO performs simulations of vehicle movements in real word maps adhering to multiple lanes, speed limits and traffic lights.

Map Layouts: Figures 5.1 and 5.2 show the selected maps for the performance evaluation of MQOG and EMQOG respectively. Both map datas were exported from OpenStreetMap Project[54]. The map data includes all roads attributes such as speed limits, lanes count, stop signs, road type, and driving direction. I chose different maps since EMQOG is testing for mobility and I needed to include the 417 highway where cars can reach up to $110 \mathrm{~km} / \mathrm{hr}$. The following maps were then converted to xml files to be processed by SUMO using NETCONVERT[55] application. Table 5.1 shows parameters for the selected maps.

Table 5.1: Map Areas

\begin{tabular}{ll}
\hline Protocol & Map Area \\
\hline MQOG & $4 \mathrm{~km} \times 3 \mathrm{~km}$ (Downtown Ottawa) \\
EMQOG & $4 \mathrm{~km} \times 3 \mathrm{~km}$ (Including 417 Highway) \\
\hline
\end{tabular}

Mobility Traces: SUMO distinguishes between a trip and a route. A trip is a vehicle movement from one place to another defined by the starting edge (street), the destination edge, and the departure time. A route is an expanded trip, that means, that a route definition contains not only the first and the last edge, but all edges the vehicle will pass. By using DUAROUTER[56] which is an application with SUMO, vehicle routes are computed using SUMO shortest path computation. DUAROUTER requires the use of the map xml file already generated by SUMO using NETCONVERT. Moreover, we 


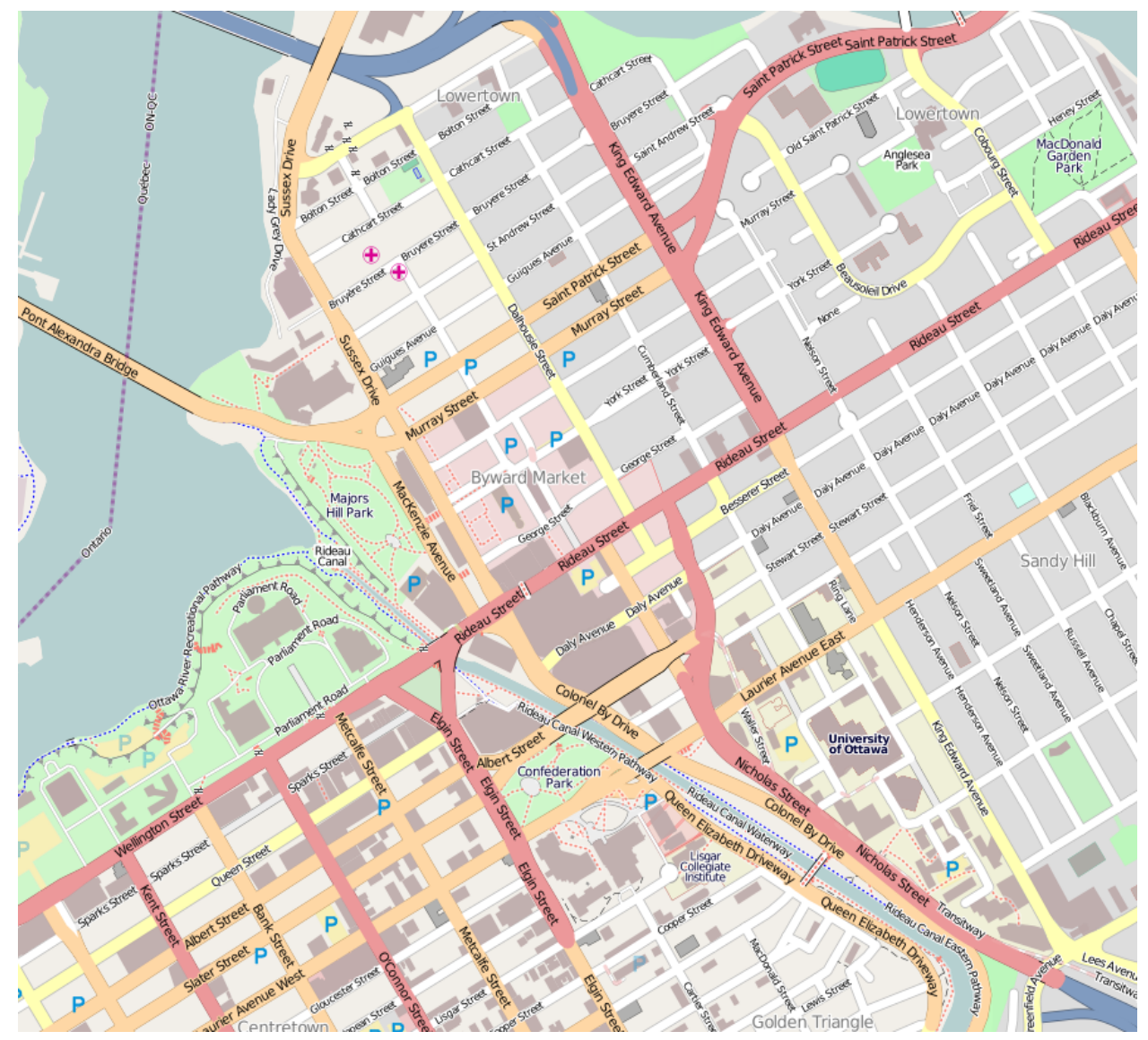

Figure 5.1: MQOG MAP for Simulation

generated many sets of trace files where each set contains traces of a specific number of vehicles. Not only we had to generate trace files when changing the number of vehicles, but also we had to generate different trace files for the same number of vehicles as it would result in more accuracy when analyzing our results. In the traces, vehicle routes are defined, where every vehicle departs at a specific simulation time in a random residential area, and goes through different edges to reach a final destination. Vehicle routes are computed using the shortest path computation, which takes into account street length, speed limits, lane count, and street type. Each trace file captures vehicles movements in the city within 15 minutes. Moreover to increase the accuracy of our model, buildings and other obstructions were added by using POLYCONVERT[57] application in SUMO. 


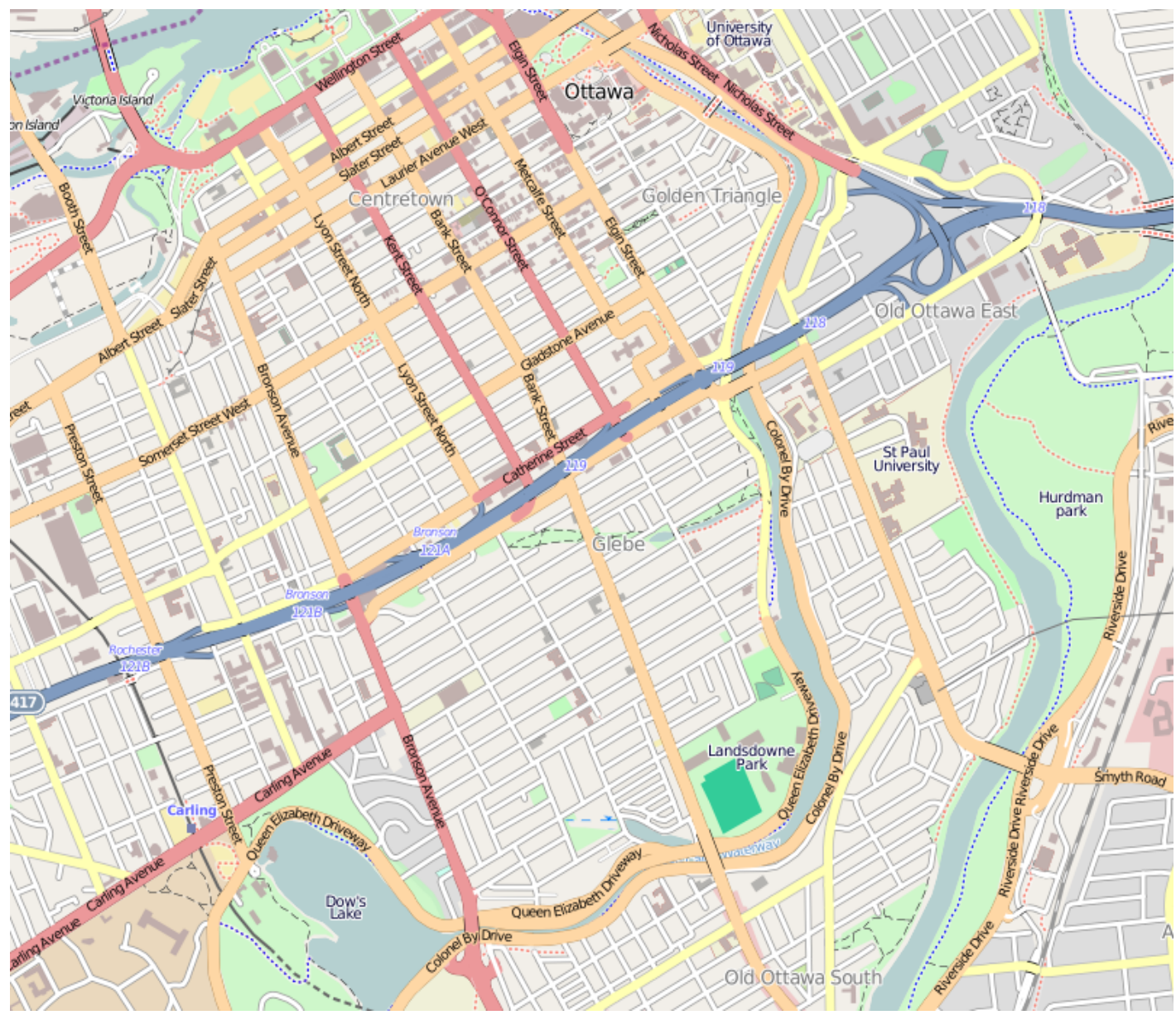

Figure 5.2: EMQOG MAP for Simulation

Car-Following Model:For SUMO parameters, we have to set a Car-Following Model. The model developed by Krauss in 1998 is a microscopic, space-continuous, car-following model based on the safe speed paradigm: A driver tries to stay away from the driver in his front at a distance and a safe speed that allows him to adapt to his leader's deceleration[58]. The model assumes the driver to have a reaction time $\tau$ of about one second. 
The model uses the following parameters[58]:

- A: the maximum acceleration of the vehicle

- B: the maximum deceleration of the vehicle

- Vmax: the maximum velocity of the vehicle

- $\mathrm{l}$ : the length of the vehicle

- e: the driver's imperfection in holding the safe speed (between 0 and 1)

- Vsafe: Safe velocity computed using the following equation:

$$
\operatorname{Vsafe}(t)=V_{L}(t)+\frac{g(t)-V_{L}(t) \tau}{\frac{V}{B \times V}+\tau}
$$

Where:

- $V_{L}(t)$ : Speed of the Leading Vehicle in time $\mathrm{t}$

- $\mathbf{g}(\mathbf{t})$ : Gap to the Leading Vehicle in time $\mathrm{t}$

- $\tau$ : The Driver's Reaction Time (usually 1s)

Traffic Control Interface (TraCI): VEINS (Vehicles in Network Simulation) [59] while using TraCI was used to bidirectionnaly couple OMNET++ with SUMO. A TCP connection is opened at port 9999 between both simulators to allow dynamic interaction during runtime. Using this port, OMNET++ and SUMO can interact with each other by sending a series of commands (e.g. speed, position... etc) during small time-steps to influence individual vehicles movements and resulted network traffic.

\subsection{Simulation Parameters, Process and Metrics}

All vehicles were configured in OMNET ++ and SUUMO to have same general simulation parameters shown in Table 5.2. In addition, we added static nodes transmitting randomly 
in the ISM and UNII-3 band to add interference on those bands which would render our simulation more realistic.

Table 5.2: General Simulation Parameters

\begin{tabular}{ll}
\hline Simulation Parameters & Values \\
\hline Radio Propagation & Nakagami \\
Radio Range & $700 \mathrm{~m}$ \\
Carrier Frequency & $5.8125-5.925 \mathrm{GHz}$ (Dynamic) \\
MAC bitrate & $12 \mathrm{Mbps}$ \\
Radio Transmit Power & $20 \mathrm{dBm}(100 \mathrm{~mW})$ \\
Vehicular Speed & Vary from $30 \mathrm{~km} / \mathrm{h}-110 \mathrm{~km} / \mathrm{hr}$ \\
Radio Sensitivity & $-85 \mathrm{dBm}$ \\
Data Frame Size & $1000 \mathrm{Bytes}$ \\
Control Frame Size & $50 \mathrm{Bytes}$ \\
Broadcast Traffic Rate & $1 \mathrm{frame} / \mathrm{s}$ \\
Broadcast Interval & $500 \mathrm{~ms}$ \\
Beacon Frequency & $1 \mathrm{~Hz}$ \\
Simulation Time & $15 \mathrm{minutes}$ \\
\hline SUMO Vehicle Parameters & Value \\
\hline Vehicle Acceleration & $2.5 \mathrm{~m} / \mathrm{s}^{2}$ \\
Vehicle Deceleration & $4.6 \mathrm{~m} / \mathrm{s}^{2}$ \\
Vehicle Length & $5 \mathrm{~m}$ \\
\hline Daximum Vehicle Speed & Varying Depending on Simulation \\
\hline
\end{tabular}

In order to quantitatively evaluate our proposed protocols, we assess different performance metrics and we compare our protocol with the IEEE 802.11p [30,34] and DCA[37] 
for MQOG and IEEE 802.11p and IEEE802.11e[31] for EMQOG. Each simulation was carried out more than 10 times and the results obtained were statistically analyzed by computing the mean and the standard deviation. On the different graphs represented, we present the mean of the collected data and the $95 \%$ Confidence Interval. The performance evaluation metrics used are the Delay, Packet Delivery Ratio, Average Throughput, and L2 Retransmission Rate.

\subsection{Performance Evaluation of the Proposed Multi- channel MAC}

In this section, I explain the simulation scenario of MQOG and the results obtained.

\subsubsection{Simulation Scenario}

To test for the efficiency of the proposed protocol in terms of the different metrics, we vary the number of vehicles in this simulation starting from 30 vehicles/area to 200 vehicles/area. Quality metrics are recorded as we are increasing the number of vehicles. We consider a communication scenario where there exists a safety broadcast and unicast transmissions. For the Safety Broadcast, we assume an accident occurred and a vehicle is broadcasting safety packets to neighboring vehicles. The traffic rate is set to 1 frame/s with a Broadcast Interval of 500ms. Simultaneously, we consider Unicast Transmissions with $30 \%$ of the vehicles in the region transmitting various QoS unicast packets to the other $30 \%$. The traffic rate is set at 1 frame/s. 


\subsubsection{Results and Interpretation}

Delay of Safety Message Reception:We evaluate the delay in ms for the reception of safety messages in the broadcast scenario. In general, signals received with high power have better transmission rate leading to a lower delay. As can be seen in Fig.5.3, the delay for all implemented protocols starts low and increases with an increasing number of vehicles. For 802.11 , we can see that at the beginning it was performing well however when the number of vehicles increases, the interference increases degrading the performance. The proposed protocol MQOG outperforms 802.11p when the number of vehicles is above 96 . As the protocol is sensing the channel and testing its transmission conditions, it is ensuring a lower delay with a higher reliability of transmission.

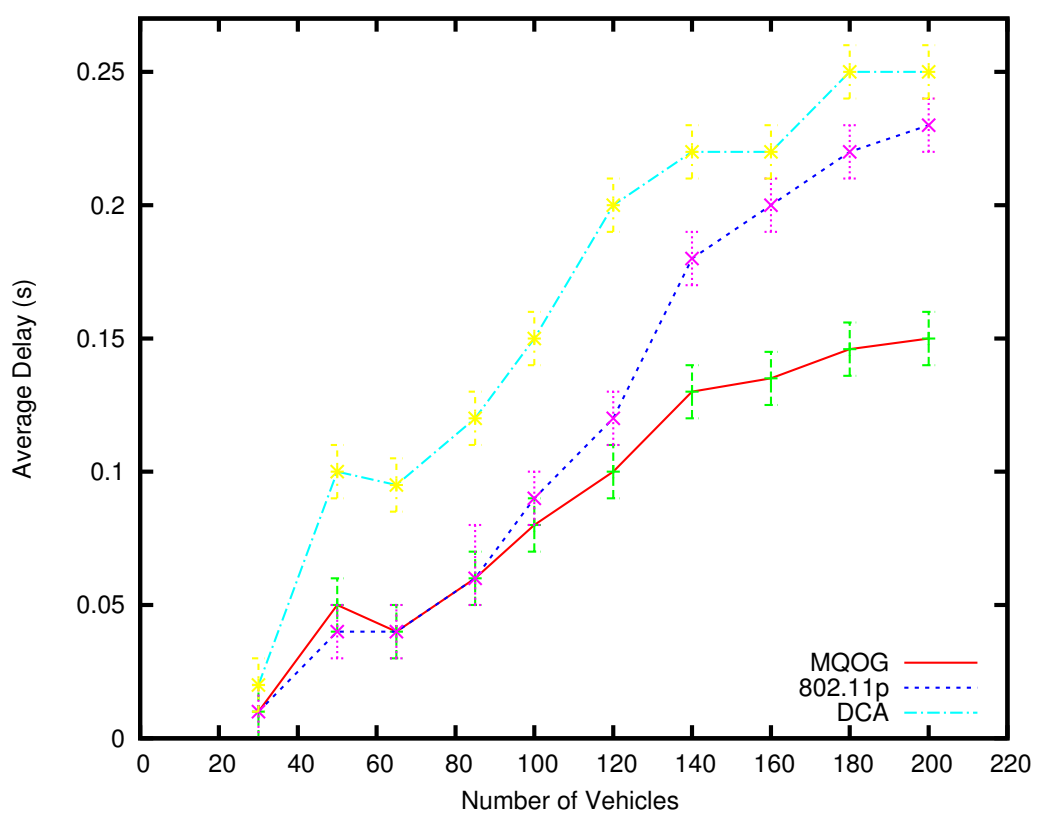

Figure 5.3: Delay of Safety Message Reception 
Packet Delivery Ratio for Safety Messages:PDR shows the ratio of number of safety messages received to the number delivered. As can be seen in Fig.5.4, 802.11p and DCA start by having a high PDR when the number of vehicles is around 80 . However as the number of vehicles increases the PDR degrades gradually. The proposed protocol MQOG outperforms 802.11p and DCA since it is ensuring the reliable transmission of safety messages by channel assessing prior to transmission. Moreover, MQOG requests a data channel to handoff to an ISM or UNII-3 channel in case there wasn't any reliable DSRC Channel. This ensures the reliable delivery of Safety messages over non safety messages.

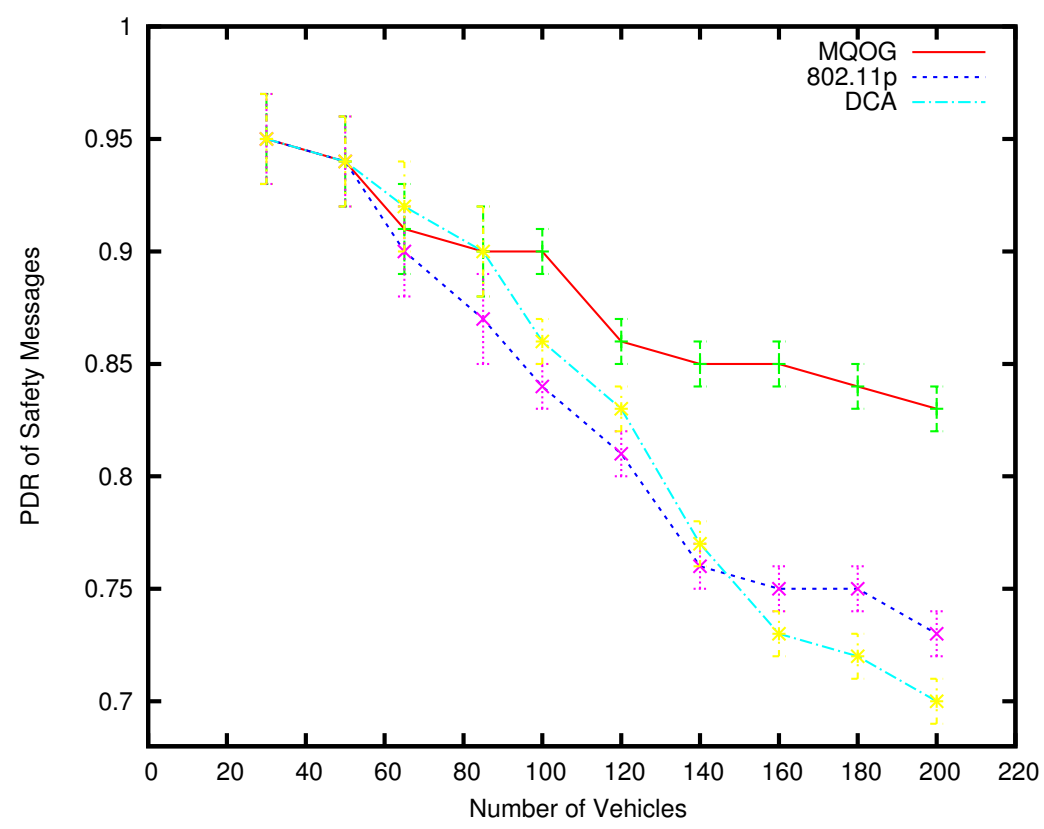

Figure 5.4: Packet Delivery Ratio for Safety Messages 
Throughput: The throughput reflects the system's performance. As shown in Fig.5.5, the throughput of MQOG starts as being slightly less than the 802.11p and DCA where the traffic generation is relatively low compared to an urban environment. This is due to the overhead that MQOG imposes on the dedicated control channel which doesn't allow it to outperform others. However as we are increasing the number of vehicles in the same area, MQOG outperforms others since it is taking into account the level of interference and compensating for them by switching to other frequencies with less interference. This switch would decrease the retransmission of corrupted packets leading to an increased throughput.

In Fig.5.6, we set the number of vehicles to 100 and we varied the unicast traffic rate generated within different cars. As can be seen, the proposed protocol outperforms others as the traffic generated is increasing. This is because the proposed protocol allows short messages to be sent on the control channel leading to better access medium contention between vehicles while long frames are sent on other channels without medium contention.

Regarding the overhead, MQOG while using the dynamic channel negotiation protocol uses the same number of overhead messages as the IEEE802.11p. The latter uses the RTS-CTS frames as part of the collision avoidance mechanism to address the hidden node problem. However, MQOG uses the adjusted RTS-CTS frames (discussed in section 3.3.2) which present the same number of frames with adjusted parameters and sizes. 


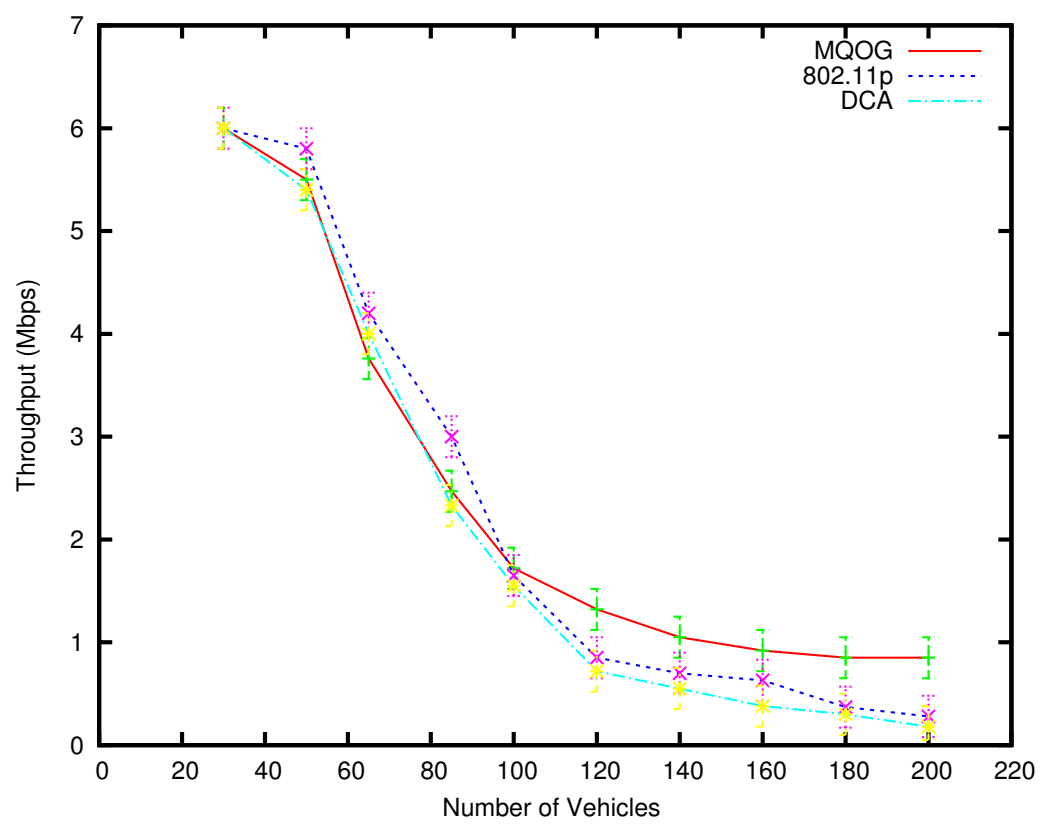

Figure 5.5: Throughput $\mathrm{x}$ Number of Vehicles

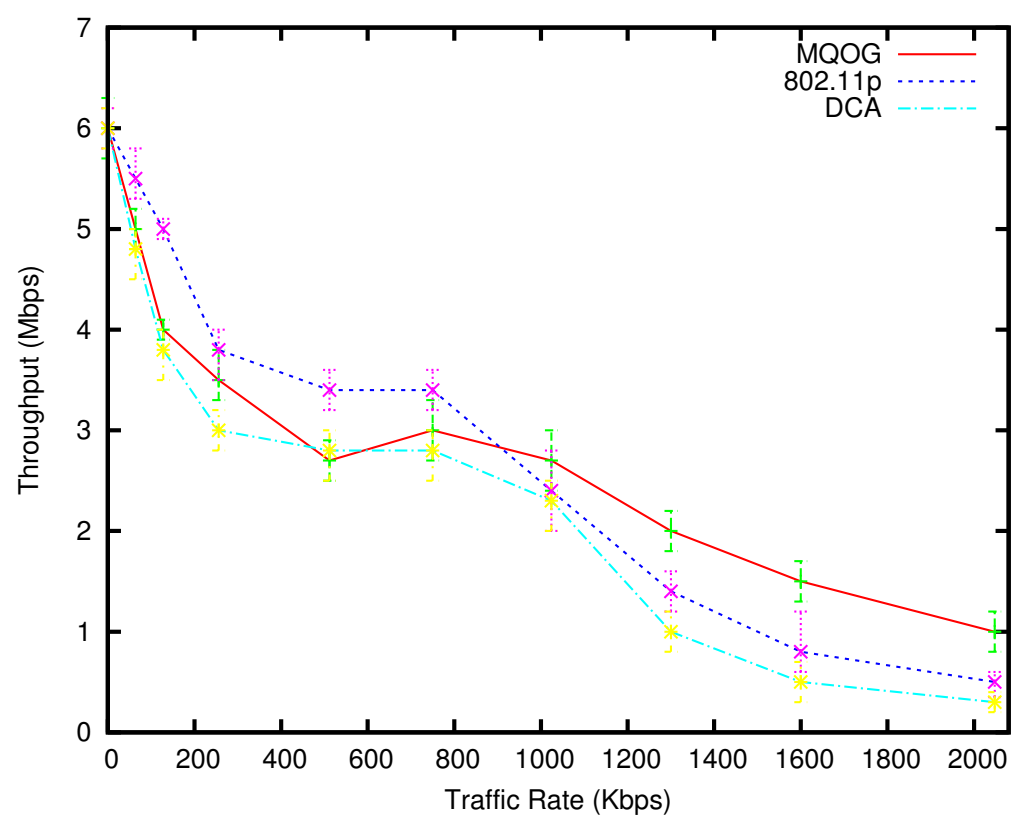

Figure 5.6: Throughput x Traffic Rate 
L2 Retransmission Rate: L2 Retransmission Rate is a direct consequence of multipath, interference and packet collisions. Fig.5.7 shows that the percentage of L2 retransmissions dropped significantly when MQOG was used instead of 802.11p or DCA. This is due to the fact that the proposed protocol takes into consideration the interference and noise level on a channel prior to transmission.

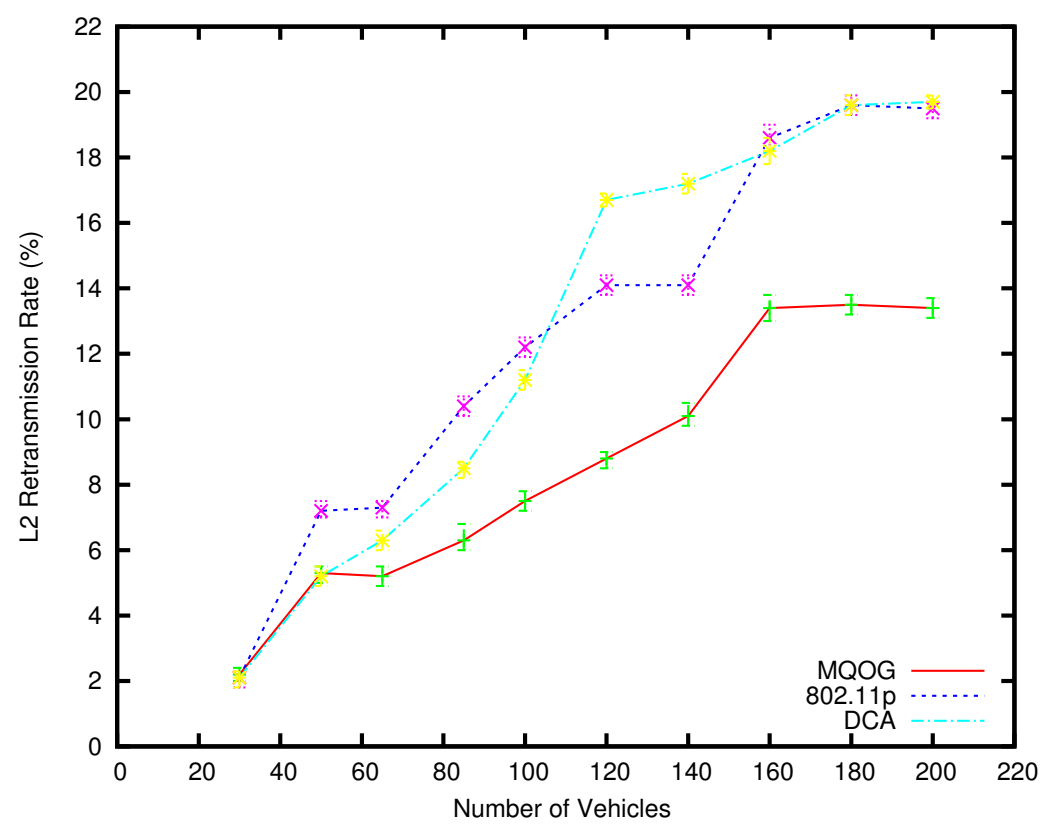

Figure 5.7: L2 Retransmission Rate

Although with low number of vehicles, MQOG doesn't provide significant advantage compared to others however its contribution lies in granting safety messages priority in transmission. Nevertheless, MQOG drastically improves throughput in congested environments by using a unique multichannel operation as it assesses channel conditions before transmission. 


\subsection{Performance Evaluation of the Proposed Enhanced Multichannel MAC}

For the EMQOG situation a map was chosen which includes the 417 highway. IEEE 802.11p is chosen for comparison and IEEE 802.11e is also selected since it is the standard with TXOP mechanism that supports multimedia communications. To evaluate the latency of frame reception for safety messages, delay was measured as the first metric. For mobility assessment, the packet delivery ratio was tracked while Cars were moving with high speeds. Moreover, we initiated various applications with different bit rates on the transmitter side while measuring the average throughput as the third metric. All those metrics were calculated in both scenarios covering the scalability of a VANET in both light and heavy load conditions. Those metrics show the efficiency of the system in reception of safety messages within time constraints, reliability of delivered frames within different speeds and system overall performance in throughput assessment.

\subsubsection{Simulation Scenario}

The two scenarios used in this assessment are A and B, presented in Table 5.3. The main difference between both scenarios is increasing the number of vehicles and thus creating an urban environment where there exists more contention on the medium. Quality metrics are recorded during simulation time for both scenarios. The metrics are being collected for a unicast 1 hop link between two cars transmitting and receiving frames. The two communicating vehicles start in the same position then drive in random directions. Other vehicles are flooding a broadcast message which was initiated by one vehicle and then disseminated throughout the network. In addition, all vehicles transmit a beacon every 1s. The beacon contains the vehicle ID, Speed, Position and direction angle $\alpha$. 
Table 5.3: Scenario Types

\begin{tabular}{llr}
\hline & Scenario A & Scenario B \\
\hline Number of Vehicles & 120 vehicles & 780 vehicles \\
Vehicular Density & 10 vehicles $/ \mathrm{km}^{2}$ & 65 vehicles $/ \mathrm{km}^{2}$ \\
Environment & Suburban & Urban \\
\hline
\end{tabular}

\subsubsection{Results and Interpretation}

Average Delay for Safety Message Reception: First, we evaluate the delay in ms for the reception of safety messages. In general, safety messages have latency constraints and should be received within short delays. As can be seen in Figures 5.8 and 5.9, the delay for all implemented protocols starts low and then increases with time. This is due to the flooding of broadcast messages between adjacent vehicles creating a wider disseminated circle. For scenario A, seen in Figure 5.8, EMQOG outperforms slightly 802.11p and $802.11 \mathrm{e}$ in 50ms. 802.11p and 802.11e have approximately very similar delays in the beginning but 802.11 e performs better after 11 minutes because of its TXOP operation that combats congested media.

On the other hand, it can be clearly seen in Figure 5.9 (urban environment) that EMQOG outperforms 802.11p and 802.11e in 100ms. As EMQOG is granting longer TXOP for safety messages, it ensures a lower delay with a higher reliability of transmission. This was clearly demonstrated when there was traffic since safety messages are prioritized over regular data traffic and by using the dynamic TXOP, traffic is being sent consecutively. In brief, EMQOG gained the edge especially in congested environments (urban) as it conveys a prioritization system and a dynamic TXOP that ensures the transmission and reception of safety messages much faster than 802.11p and 802.11e MAC protocols. 


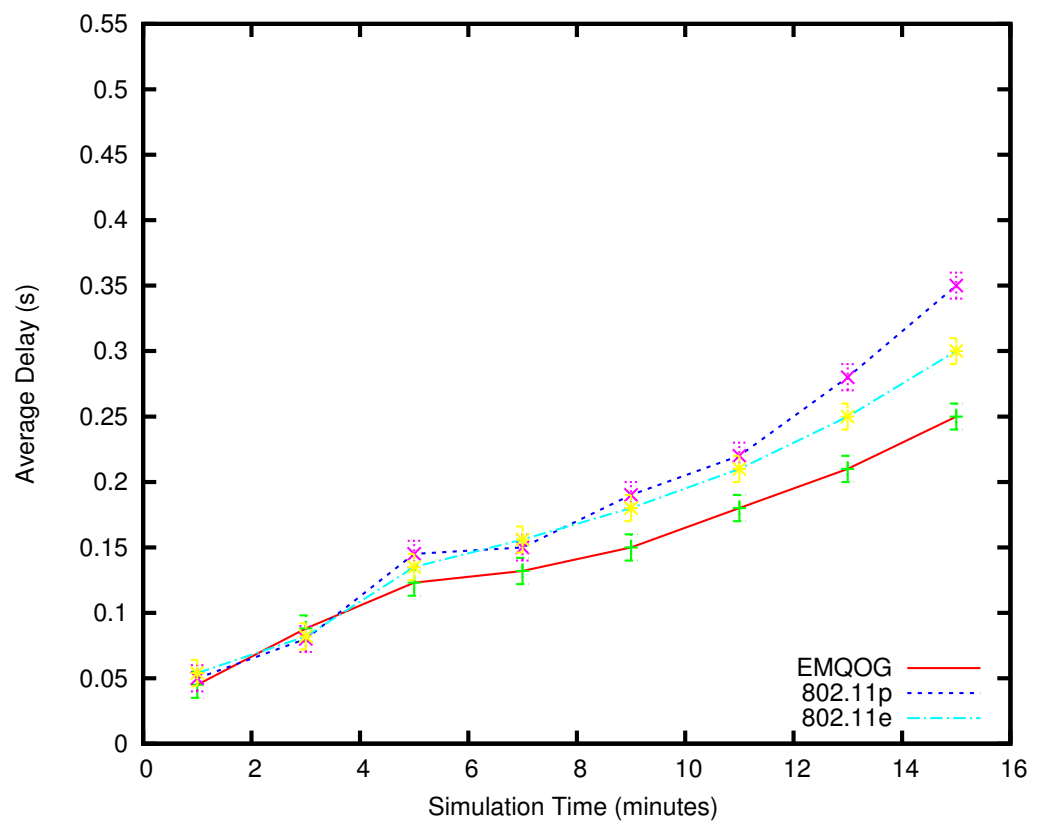

Figure 5.8: Average Delay for Safety Message Reception - Scenario A

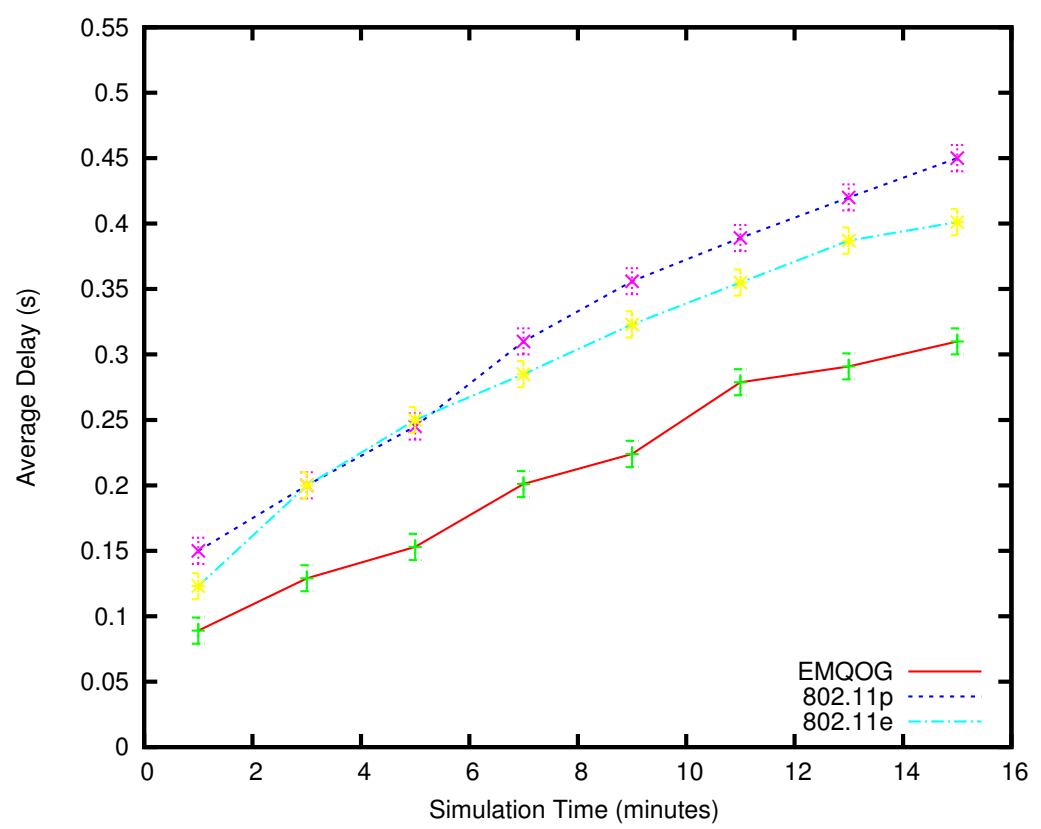

Figure 5.9: Average Delay for Safety Message Reception - Scenario B 
General Packet Delivery Ratio (PDR): To assess the impact of mobility, we evaluate the PDR (Packet Delivery Ratio) while varying the cars' speeds. As SUMO sets the speed limits on the downloaded map roads and cars, we alter the maximum car speed in the Krauss model to an upper threshold however cars drive within speed limits. This speed is set in our simulation and it represents the x-component of our graph. The simulation is repeated each time with a different maximum speed to evaluate the performance in different mobility conditions. In general, PDR represents the ratio of number of messages received to the number delivered. As can be seen in Figure 5.10 (Scenario A), all MAC protocols start by having a high PDR with low speeds. However as the vehicular speed increases (above $70 \mathrm{~km} / \mathrm{hr}$ ), the PDR drops gradually due to high mobility impact which is not considered in 802.11e. 802.11p performs better than 802.11e as it has mobility consideration but lacks the dynamic TXOP operation incorporated in EMQOG.

On the other hand, in the urban environment which can be seen in Figure 5.11, as the medium contention increases, EMQOG much outperforms 802.11e and 802.11p. This is because it considers vehicle's speeds while allocating the necessary TXOP limit. Hence, vehicles driving in high speeds would have a longer TXOP limit allocating the medium for a longer time and submitting a longer frame burst. EMQOG outperformed others especially in congested environments with cars driving in high speeds as it is designed to mitigate mobility and congestion unlike other MAC layer protocols. 


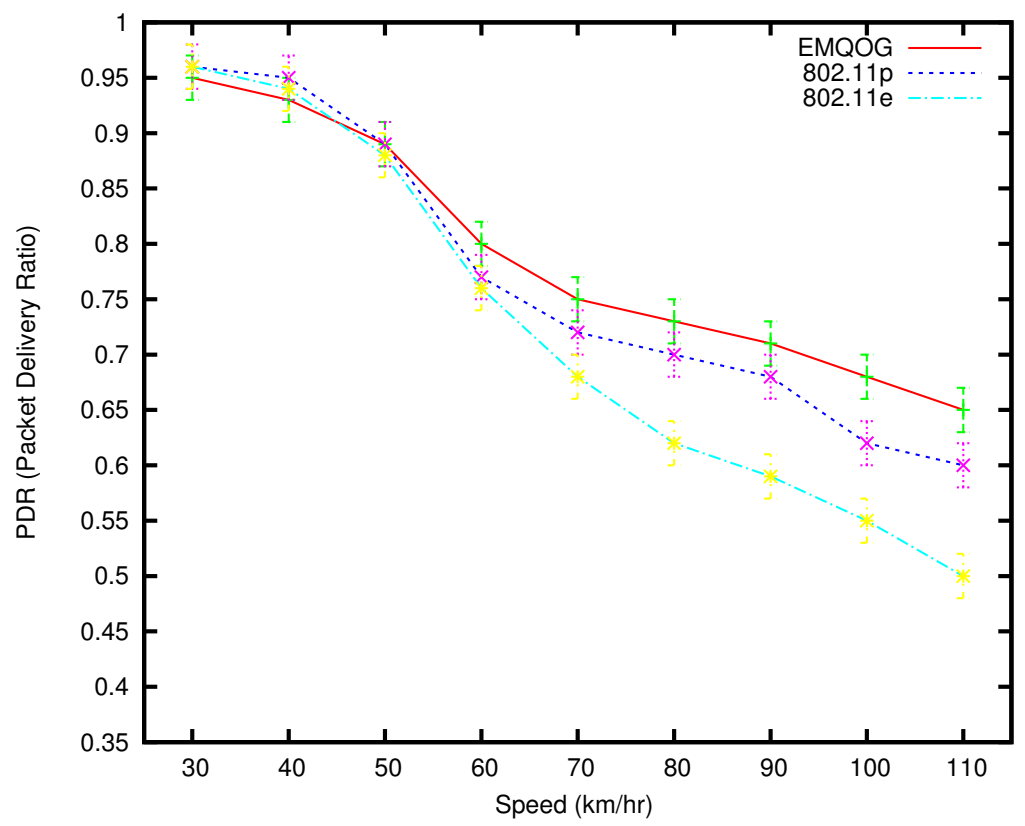

Figure 5.10: Packet Delivery Ratio - Scenario A

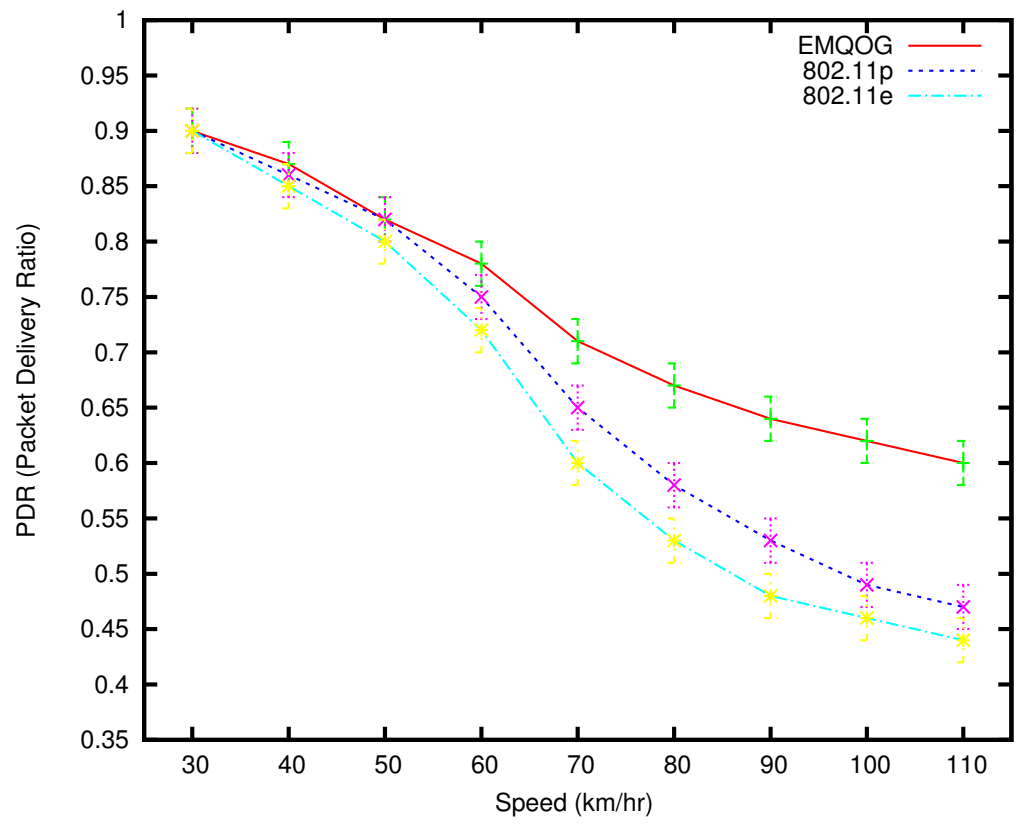

Figure 5.11: Packet Delivery Ratio - Scenario B 
Average Throughput: The average throughput reflects the system's overall performance. As shown in Figures 5.12 and 5.13, there wasn't much difference in the simulation results for both 802.11e and 802.11p. On the other hand, EMQOG outperformed them in both scenarios A and B. This is due to the fact that EMQOG is transmitting a frame burst with short delays hence enhancing the throughput achieved. As we are shifting from a suburban to an urban environment i.e. from scenario A to scenario B, EMQOG performed much better than the others since it considers the transmit data rate whereas 802.11p and 802.11e overlook that aspect in the MAC layer. Moreover, EMQOG considers the average data rate and forces vehicles to cooperate fairly in the contention process. In addition, as the traffic generated is increasing, the throughput is degrading because of the excessive medium contention. 802.11e and 802.11p are not efficient when the traffic increases since they lack smart mechanisms for medium contention on the MAC layer. This is the reason why EMQOG outperformed them in congested scenarios. 


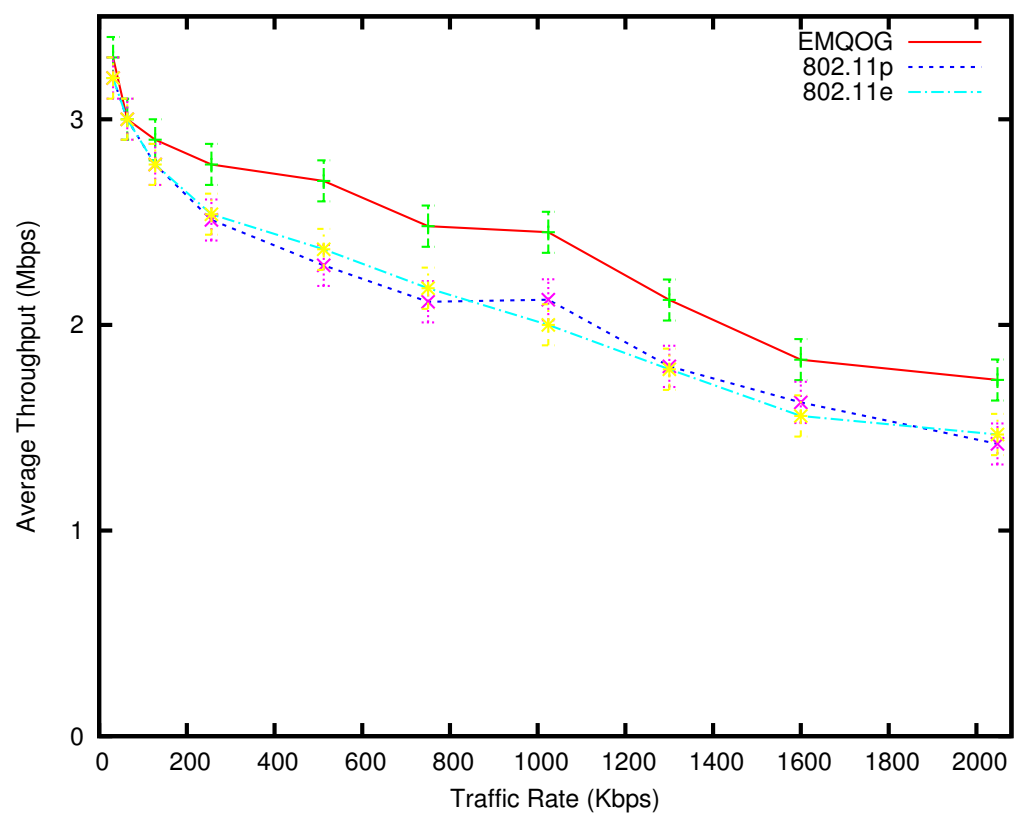

Figure 5.12: Average Throughput x Traffic Rate - Scenario A

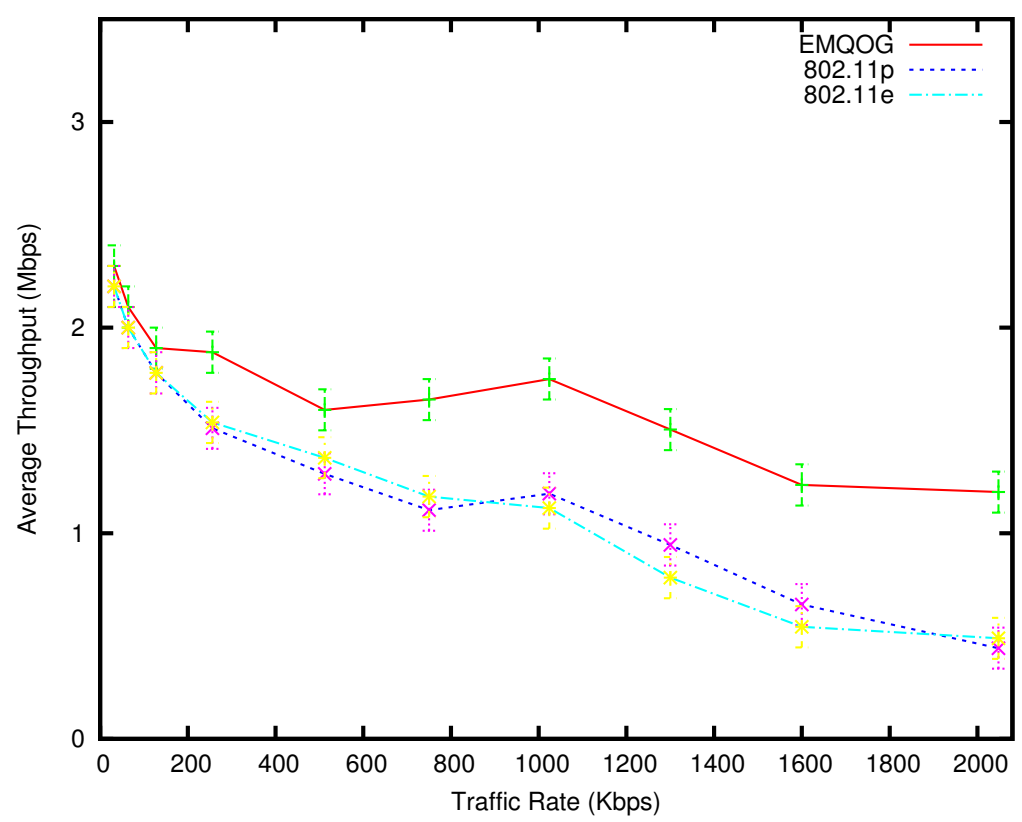

Figure 5.13: Average Throughput x Traffic Rate - Scenario B 
Overall, EMQOG outperformed 802.11p and 802.11e and is more suitable for VANet environments with varying traffic conditions. Although with low medium contention, EMQOG doesn't provide an edge compared to others however its contribution lies in considering mobility, prioritizing safety messages, and ensuring a better throughput. Moreover, EMQOG major contribution is in congested environments where medium contention is an issue as it smartly allocates the medium based on mobility, QoS, and data rate considerations.

\subsection{Conclusion}

To evaluate the performance of MQOG and EMQOG, implementation was done in OMNet++ 4.1 and our results showed an improvement in delay, packet delivery ratio, throughput, and L2 retransmissions. Simulation results demonstrated that unlike 802.11p contention based protocols, DCA channel negotiation style protocols, or 802.11e with static TXOP, MQOG can offer better vehicle safety through smaller latency and less L2 retransmission rates, better efficiency in performance through an increased throughput and a better reliability through an increased packet delivery ratio. It has also been shown that the proposed protocol and mechanism are more suitable to be used under urban and congested environments instead of suburban ones. 


\section{Chapter 6}

\section{Conclusion and Future Work}

Due to the inherent characteristics and diverse challenges facing vehicular networks, existing MAC protocols are not tailored to meet VANet's requirements. In this thesis, a new multichannel MAC protocol is introduced MQOG followed by an enhacement mechanism MoByToP to improve the performance of MAC contention and reservation in vehicular networks. The proposed protocol together with the enhancement mechanism were both evaluated with OMNET ++ and simulations showed improvement for the MAC in throughput, delay, Packet Delivery Ratio, and L2 retransmissions.

First, a background discussion of MAC protocols is presented. Moreover, MAC design guidelines were highlighted. Following a top down approach, diverse multiple access schemes were explained and then the most suitable protocols for VANets were discussed. From the perspective of using different MAC architectures, overviews of the existing MAC solutions for a vehicular environment were briefly introduced. This provided a broad view of the current existing MAC protocols for VANets. Finally, a qualitative comparison between different existing protocols was provided.

I then proposed a new Multichannel QoS Cognitive MAC (MQOG) tailored for vehicular communications. MQOG ensures QoS by granting safety messages higher priority 
of accessing the medium over data messages by making use of the ISM and UNII bands which precede the DSRC Band. Moreover, MQOG assesses the channel prior to transmission messages are always sent on the best available channel to mitigate high interference and multipath problems. Those features make the proposed protocol MQOG more suitable for VANET environments.

Moreover, I propose an Enhanced MQOG which is a new MAC enhancement mechanism added on top of MQOG. MoByToP, a MAC Layer mechanism, tailored for vehicular communications incorporates mobility, QoS and transmit data rate and integrates them into the MAC to mitigate VANet challenges. To best of my knowledge, I am the first to propose a dynamic transmit opportunity to alleviate the fairness issue in vehicular environments. MoBytoP uses beacon information to calculate LOP (Link Out of Range Prediction) and then uses it to circumvent link failure. In addition, it adjusts the frame burst based on the RSS (Received Signal Strength) to create a fair effective sharing of resources and hence enhancing the system's overall throughput. Moreover, it ensures QoS by granting safety messages higher priority of reserving the medium over data messages.

To evaluate the performance of MQOG and EMQOG, implementation and simulation were carried out in OMNet++ 4.1 and my results showed an improvement in throughput, delay, packet delivery ratio, and L2 retransmissions. Moreover, it has also been shown that the proposed protocol and mechanism were more suitable to be used under urban and congested environments instead of suburban ones.

\subsection{Future Work}

Although this thesis provides a comprehensive study and evaluation from different perspectives, there are still some open issues and several research directions that can be pursued to improve the performance of MQOG and MoByToP. 
- Multiple Hops: The proposed MAC protocol MQOG and the enhancement mechanism MoByToP are both for 1 hop transmission. Future work may expand the operation of both of them to consider multiple hops.

- Heterogeneous Networks: Work can be done to provide a MAC handoff process between different heterogeneous networks such as cellular networks or WLANs from one side and vehicular networks from the other. Moreover, the MQOG architecture of having multiple channel can be helpful in the handoff process and may facilitate such convergence implementations.

- Fuzzy adaptiveness: It is very difficult to quantify speeds in real world environments as they are always fluctuating. There is always a fuzzy region between the different classes of relative speed. Therefore, to avoid crisp assignment, fuzzy logic based soft computing is expected to give better quantification. 


\section{References}

[1] CNN Money: "Bill Ford looks ahead". May 17, 2011. http://management.fortune.cnn.com/2011/05/17/bill-ford-looks-ahead/.

[2] World Health Organization(WHO):

http://www.who.int/violence_injury_prevention/road_traffic/en/

[3] Toor, Y.; Muhlethaler, P.; Laouiti, A.; , "Vehicle Ad Hoc networks: applications and related technical issues," Communications Surveys and Tutorials, IEEE , vol.10, no.3, pp.74-88, Third Quarter 2008

[4] Jiang, D.; Taliwal, V.; Meier, A.; Holfelder, W.; Herrtwich, R.; , "Design of 5.9 ghz dsrc-based vehicular safety communication," Wireless Communications, IEEE , vol.13, no.5, pp.36-43, October 2006

[5] Mak, T.K.; Laberteaux, K.P.; Sengupta, R.; Ergen, M.; , "Multichannel Medium Access Control for Dedicated Short-Range Communications," Vehicular Technology, IEEE Transactions on , vol.58, no.1, pp.349-366, Jan. 2009

[6] Hongyi Wu, Yi Pan (2008). Medium Access Control in Wireless Networks. Nova Scotia: Nova Science Pub Inc. 120-123.

[7] Alex Brand, Hamid Aghvami (2002). Multiple Access Protocols for Mobile Communications: GPRS, UMTS and Beyond. New York: Wiley. 
References

[8] David D. Coleman, David A. Westcott (2009). CWNA Certified Wireless Network Administrator Official Study Guide. Sybex John Wiley and Sons McGraw-Hill

[9] "IEEE Standard for Information Technology-Telecommunications and Information Exchange Between Systems-Local and Metropolitan Area Networks-Specific Requirements - Part 11: Wireless LAN Medium Access Control (MAC) and Physical Layer (PHY) Specifications," IEEE Std 802.11-2007 (Revision of IEEE Std 802.111999) , vol., no., pp.C1-1184, June 122007

[10] S. V. Bana and P. Varaiya, "Space division multiple access (SDMA) for robust ad hoc vehicle communication networks," in Intelligent Transportation Systems, 2001. Proceedings. 2001 IEEE, 2001, pp. 962-967.

[11] S. Katragadda, C. N. S. Ganesh Murthy, M. S. Ranga Rao, S. Mohan Kumar, and R. Sachin, "A Decentralized Location-Based Channel Access Protocol for Intervehicle Communication," in The 57th IEEE Semiannual Vehicular Technology Conference, 2003, pp. 1831-1835, vol.3.

[12] R. Mangharam, R. Rajkumar, M. Hamilton, P. Mudalige, and F. Bai, "BoundedLatency Alerts in Vehicular Networks," in Mobile Networking for Vehicular Environments, 2007, pp. 55-60.

[13] J. B. Jeremy and E. Azim, " A Reliable Link-Layer Protocol for Robust and Scalable Intervehicle Communications," IEEE Transactions on Intelligent Transportation Systems, vol. 8, pp. 4-13, 2007.

[14] H. Nakata, T. Inoue, M. Itami, and K. Itoh, "A Study of Inter Vehicle Communication Scheme Allocating PN Codes to The Location On The Road," in Proc.of IEEE Intelligent Transportation Systems, 2003, pp. 1527-1532, vol.2. 
References

[15] C. Xianbo and H. H. Refai, "SDMA: On The Suitability for VANET," in 3rd International Conference on Information and Communication Technologies: From Theory to Applications, 2008, pp. 1-5.

[16] F. Borgonovo, A. Capone, M. Cesana, and L. Fratta. ADHOC: a new, exible and reliable MAC architecture for ad-hoc networks. IEEE Wireless Communications and Networking, pages 965970. v. 2, 2003.

[17] The European Project CarTALK 2000: http://www.cartalk2000.net/

[18] H. Menouar, F. Filali, and M. Lenardi. A survey and qualitative analysis of mac protocols for vehicular ad hoc networks. IEEE Wireless Communications, 13(5)

[19] X. Chen, H.H. Refai, and X. Ma. On the enhancements to IEEE 802.11 MAC and their suitability for safety-critical applications in VANET. Wireless Communications and Mobile Computing, 2008.

[20] C. Cuyu, Y. Xiang, S. Meilin, and L. Lin. Performance Observations on MAC Protocols of VANETs in Intelligent Transportation System. International Conference on Communications and Mobile Computing, pages 373.

[21] Q. Xu, T. Mak, J. Ko, and R. Sengupta. Vehicle-to-vehicle safety messaging in DSRC. In Proceedings of the 1st ACM international workshop on Vehicular ad hoc networks, pages 19

[22] Q. Xu, T. Mak, J. Ko, and R. Sengupta. Medium Access Control Protocol Design for Vehicle Vehicle Safety Messages. IEEE Transactions on Vehicular Technology, 56(2):499,518, 2007.

[23] F. Farnoud, B. Hassanabadi, and S. Valaee. Message Broadcast using Optical Orthogonal Codes in Vehicular Communication Systems. Wireless Networking for Intelligent Transportation Systems Workshop, 2007. 
References

[24] F. Farnoud and S. Valaee. Repetition-based broadcast in vehicular ad hoc networks in Rician channel with capture. IEEE INFOCOM Workshops, pages 1, 6, 2008.

[25] B. Hassanabadi, L. Zhang, and S. Valaee. Index Coded Repetition-Based MAC in Vehicular Ad-Hoc Networks. IEEE Consumer Communications and Networking Conference, pages 1,6, 2009.

[26] R.M. Yadumurthy, A.C. H., M. Sadashivaiah, and R. Makanaboyina. Reliable MAC broadcast protocol in directional and omni-directional transmissions for vehicular ad hoc networks. In Proceedings of the 2nd ACM international workshop on Vehicular ad hoc networks, pages 10,19, 2005.

[27] X. Xie, F. Wang, H. Wang, and K. Li. Adaptive Multi-channel MAC Protocol for Dense VANET Using Directional Antennas. Second International Conference on Future Generation Communication and Networking, pages 398,401. v. 2, 2008.

[28] K.-T. Feng. LMA: Location- and Mobility-Aware Medium-Access Control Protocols for Vehicular Ad Hoc Networks Using Directional Antennas. IEEE Transactions on Vehicular Technology, 56(6):3324,3336, 2007.

[29] H. Dai, K.-W. Ng, and M.-Y. Wu. An Overview of MAC Protocols with Directional Antennas in Wireless ad hoc Networks. International Conference on Wireless and Mobile Communications, pages 84, 84, 2006.

[30] 802.11p-2010 - IEEE Standard for Information technology-Telecommunications and information exchange between systems-Local and metropolitan area networksSpecific requirements Part 11: Wireless LAN Medium Access Control (MAC) and Physical Layer (PHY) Specifications Amendment 6: Wireless Access in Vehicular Environments 
References

[31] "IEEE Standard for Information Technology - Telecommunications and Information Exchange Between Systems - Local and Metropolitan Area Networks - Specific Requirements Part 11: Wireless LAN Medium Access Control (MAC) and Physical Layer (PHY) Specifications Amendment 8: Medium Access Control (MAC) Quality of Service Enhancements," IEEE Std 802.11e-2005 (Amendment to IEEE Std 802.11, 1999 Edition (Reaff 2003), vol., no., pp.0 1-189, 2005)

[32] Molisch, A.; Tufvesson, F.; Karedal, J.; Mecklenbrauker, C.; , " A survey on vehicleto-vehicle propagation channels," Wireless Communications, IEEE , vol.16, no.6, pp.12-22, December 2009

[33] Jiang, D.; Delgrossi, L.; , "IEEE 802.11p: Towards an International Standard for Wireless Access in Vehicular Environments," Vehicular Technology Conference, 2008. VTC Spring 2008. IEEE, vol., no., pp.2036-2040, 11-14 May 2008

[34] 1609.4-2010 - IEEE Standard for Wireless Access in Vehicular Environments (WAVE)- Multi-channel Operation

[35] Yiu, W.-P.K.; Xing Jin; Chan, S.-H.G.; , "VMesh: Distributed Segment Storage for Peer-to-Peer Interactive Video Streaming," Selected Areas in Communications, IEEE Journal on , vol.25, no.9, pp.1717-1731, December 2007

[36] Hang Su; Xi Zhang; , "Clustering-Based Multichannel MAC Protocols for QoS Provisionings Over Vehicular Ad Hoc Networks," Vehicular Technology, IEEE Transactions on , vol.56, no.6, pp.3309-3323, Nov. 2007

[37] Shih-Lin Wu; Chih-Yu Lin; Yu-Chee Tseng; Jang-Laing Sheu; , "A new multichannel MAC protocol with on-demand channel assignment for multi-hop mobile ad hoc networks," Parallel Architectures, Algorithms and Networks, 2000. I-SPAN 2000. Proceedings. International Symposium on , vol., no., pp.232-237, 2000 
References

[38] Zhao, Q; Sadler,B, "A Survey of Dynamic Spectrum Access: Signal processing, networking, and regulatory policy," IEEE Signal Processing Magazine, 2007. Volume: 24, Issue: 3, Pages: 79-89

[39] J. Mitola, Software Radios: Wireless Architecture for the 21st Century. John Wiley and Sons Inc, 2000.

[40] Fluke Networks: AirMagnet Survey Datasheet

[41] Der-Jiunn Deng; Chih-Heng Ke; Hsiao-Hwa Chen; Yueh-Min Huang; , "Contention window optimization for ieee 802.11 DCF access control," Wireless Communications, IEEE Transactions on , vol.7, no.12, pp.5129-5135, December 2008

[42] Jianbing Mao; Yuming Mao; Supeng Leng; Xiang Bai; , "A simple adaptive optimization scheme forIEEE 802.11 with differentiated channel access," Communications Letters, IEEE , vol.13, no.5, pp.297-299, May 2009

[43] Cali, F.; Conti, M.; Gregori, E.; , "Dynamic tuning of the IEEE 802.11 protocol to achieve a theoretical throughput limit," Networking, IEEE/ACM Transactions on , vol.8, no.6, pp.785-799, Dec 2000

[44] Ehsan Karamad and Farid Ashtiani. 2008. A modified 802.11-based MAC scheme to assure fair access for vehicle-to-roadside communications. Comput. Commun. 31, 12 (July 2008), 2898-2906

[45] Alasmary, W.; Weihua Zhuang; , "The Mobility Impact in IEEE 802.11p Infrastructureless Vehicular Networks," Vehicular Technology Conference Fall (VTC 2010Fall), 2010 IEEE 72nd, vol., no., pp.1-5, 6-9 Sept. 2010

[46] Andreadis, A.; Zambon, R.; , "QoS Enhancement with Dynamic TXOP Allocation in IEEE 802.11e," Personal, Indoor and Mobile Radio Communications, 2007. 
References

PIMRC 2007. IEEE 18th International Symposium on , vol., no., pp.1-5, 3-7 Sept. 2007

[47] Nan Guo; Chen Chen; Chang-xing Pei; , "Dynamic TXOP Assignment for Fairness (DTAF) in IEEE 802.11e WLAN under Heavy Load Conditions," Parallel and Distributed Computing, Applications and Technologies, 2006. PDCAT '06. Seventh International Conference on , vol., no., pp.80-85, Dec. 2006

[48] Majkowski, Jakub; Palacio, Ferran Casadevall; , "Dynamic TXOP configuration for Qos enhancement in IEEE 802.11e wireless LAN," Software in Telecommunications and Computer Networks, 2006. SoftCOM 2006. International Conference on , vol., no., pp.66-70, Sept. 29 2006-Oct. 12006

[49] Ghaboosi, K.; Latva-aho, M.; Yang Xiao; Khalaj, B.H.; , "IEEE 802.11 Distributed Coordination Function service time and queuing delay analysis using Parallel Space - Time Markov Chain," Personal, Indoor and Mobile Radio Communications, 2008. PIMRC 2008. IEEE 19th International Symposium on , vol., no., pp.1-5, 15-18 Sept. 2008

[50] "IEEE Standard for Information technology-Telecommunications and information exchange between systems-Local and metropolitan area networks-Specific requirements Part 11: Wireless LAN Medium Access Control (MAC) and Physical Layer (PHY) Specifications Amendment 6: Wireless Access in Vehicular Environments," IEEE Std 802.11p-2010 (Amendment to IEEE Std 802.11-2007 as amended by IEEE Std 802.11k-2008, IEEE Std 802.11r-2008, IEEE Std 802.11y-2008, IEEE Std 802.11n-2009, and IEEE Std 802.11w-2009), vol., no., pp.1-51, July 152010

[51] Paier, A.; Tresch, R.; Alonso, A.; Smely, D.; Meckel, P.; Zhou, Y.; Czink, N.; , "Average Downstream Performance of Measured IEEE 802.11p Infrastructure- 
to-Vehicle Links," Communications Workshops (ICC), 2010 IEEE International Conference on , vol., no., pp.1-5, 23-27 May 2010

[52] INETMANET Framework for OMNEST/OMNeT++ 4.x (based on INET Framework) : http://inet.omnetpp.org/

[53] Simulation of Urban Mobility (SUMO) :

http://sumo.sourceforge.net/

[54] Open Street Map Project:

http://www.openstreetmap.org/

[55] SUMO NETCONVERT APPLICATION:

http://sourceforge.net/apps/mediawiki/sumo/index.php?title=NETCONVERT

[56] SUMO DUAROUTER APPLICATION:

http://sourceforge.net/apps/mediawiki/sumo/index.php?title=DUAROUTER

[57] SUMO POLYCONVERT APPLICATION:

http://sourceforge.net/apps/mediawiki/sumo/index.php?title=POLYCONVERT

[58] RANJITKAR Prakash; NAKATSUJI Takashi; KAWAMUA Akira; , "Car Following Models: An Experiment Based Benchmarking," Journal of the Eastern Asia Society for Transportation Studies, Vol. 6, pp. 1582 - 1596, 2005

[59] Vehicles In Network Simulation (VEINS): http://veins.car2x.org/ 